\title{
Pulsed Molecular Optomechanics in Plasmonic Nanocavities: From Nonlinear Vibrational Instabilities to Bond-Breaking
}

\author{
Anna Lombardi, ${ }^{1}$ Mikołaj K. Schmidt, ${ }^{2,3,4}$ Lee Weller, ${ }^{1}$ William M. Deacon, ${ }^{1}$ \\ Felix Benz, ${ }^{1}$ Bart de Nijs, ${ }^{1}$ Javier Aizpurua, ${ }^{2}$ and Jeremy J. Baumberg ${ }^{1, *}$ \\ ${ }^{1}$ NanoPhotonics Centre, Cavendish Laboratory, \\ University of Cambridge, Cambridge CB3 OHE, United Kingdom \\ ${ }^{2}$ Materials Physics Center CSIC-UPV/EHU and Donostia International Physics Center DIPC, \\ Paseo Manuel de Lardizabal, 20018 Donostia-San Sebastián, Spain \\ ${ }^{3}$ Faculty of Physics, University of Warsaw, Pasteura 5, 02-093 Warsaw, Poland \\ ${ }^{4}$ Macquarie University Research Centre in Quantum Science and Technology (QSciTech), \\ Department of Physics and Astronomy, Macquarie University NSW 2109, Australia
}

(Received 2 February 2017; revised manuscript received 6 October 2017; published 2 February 2018)

\begin{abstract}
Small numbers of surface-bound molecules are shown to behave as would be expected for optomechanical oscillators placed inside plasmonic nanocavities that support extreme confinement of optical fields. Pulsed Raman scattering reveals superlinear Stokes emission above a threshold, arising from the stimulated vibrational pumping of molecular bonds under pulsed excitation shorter than the phonon decay time, and agreeing with pulsed optomechanical quantum theory. Reaching the parametric instability (equivalent to a phonon laser or "phaser" regime) is, however, hindered by the motion of gold atoms and molecular reconfiguration at phonon occupations approaching unity. We show how this irreversible bond breaking can ultimately limit the exploitation of molecules as quantum-mechanical oscillators, but accesses optically driven chemistry.
\end{abstract}

DOI: 10.1103/PhysRevX.8.011016

\section{INTRODUCTION}

The vibrational properties of molecules have long been exploited for analytic chemical techniques, such as infrared [1,2] and Raman spectroscopies [3-6], as well as for coherent light emission from masers [7]. The nature of chemical bonds as quantum vibrational oscillators can now be very effectively manipulated by trapping light tightly enough around molecules [8]. Analogous optomechanical systems use macroscopic vibrational modes such as cantilevers $[9,10]$, beams [11], or pillars [12-15] coupled to highfinesse optical cavities in order to demonstrate a variety of effects such as cooling to the quantum vibrational ground state [16], parametric oscillation [17], and supercontinuum comb generation [18]. Recent theory [19,20] and experiments $[8,21]$ have shown that this same optomechanical Hamiltonian describes vibrating molecular bonds in nanocavities, but with single-photon coupling coefficients

\footnotetext{
* Corresponding author. jjb12@cam.ac.uk

Published by the American Physical Society under the terms of the Creative Commons Attribution 4.0 International license. Further distribution of this work must maintain attribution to the author(s) and the published article's title, journal citation, and DOI.
}

Subject Areas: Chemical Physics, Nanophysics, Plasmonics

$\hbar g_{0} \sim 10-100 \mathrm{meV}$, a millionfold larger than for macroscopic resonators. Here, we explore how this coupling can be used to elicit stimulated phonon scattering within vibrating bonds, leading toward phonon lasing or "phasing," observed previously in microfabricated mechanical oscillators [22-24], and we suggest how such mechanical resonances can lead to chemical reactions [Fig. 1(a)].

Raman scattering that creates (Stokes) or annihilates (anti-Stokes) phonons can directly probe the phonon populations. Using an optomechanical model, it is possible to extract the photon-phonon coupling $[8,20]$. When the nanocavities are formed by ultrathin gaps between plasmonic metal nanostructures that trap the optical field, this strongly amplified process is termed surface-enhanced Raman spectroscopy (SERS) of the molecules in the gap. This process has been widely exploited for vibrational spectroscopy by enabling tagless detection of ultrasmall concentrations of chemical analytes [3,25-28]. Our understanding that molecular optomechanics and SERS correspond to the same process opens up the exploration of nonlinear Raman scattering, particularly since the welldefined plasmonic nanocavities required have now become available through self-assembly [29], lithography [30], and scanning tip microscopies [21]. We note that previous studies have been hampered by irreproducibility in the plasmonic constructs such as micropatterned substrates 
(a)
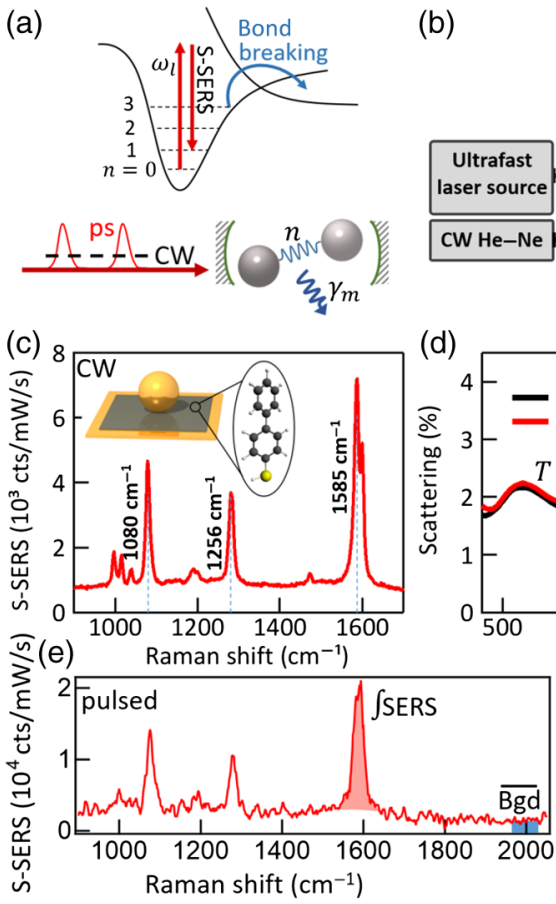

(d)

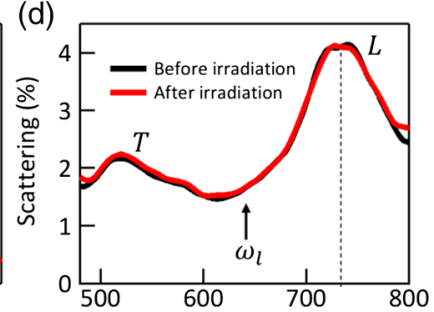

Wavelength $(\mathrm{nm})$

(f) $\bar{n} 4$ SERS

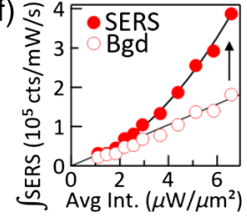

FIG. 1. Molecular optomechanics. (a) Energy diagram for ground and excited molecular vibrational states $n$ (top) pumped by laser at $\omega_{l}$, with schematic optomechanical cavity (bottom). The vibrational decay rate is $\gamma_{m}$. (b) SERS setup for singlenanoparticle studies, using continuous $(\mathrm{CW})$ or ps pulsed excitation. (c) Stokes S-SERS from single 80-nm gold nanoparticle-on-mirror (NPoM, geometry in inset), with biphenyl-4thiol (BPT) self-assembled molecular monolayer spacer. (d) Dark-field scattering from single gold NPoM before (black) and after (red) 633-nm laser irradiation with average intensity $I=2 \mu \mathrm{W} / \mu \mathrm{m}^{2}$. Coupled plasmon mode $(L)$ and transverse mode (T) resonances are marked. (e) Pulsed ps S-SERS from single NPoM, showing extraction of integrated SERS peaks and average background. (f) Power dependence of SERS (nonlinear) and background (linear) components.

[31], colloidal aggregates [32], and micron-sized particles [33]. Moreover, because phonon lifetimes in molecules are on the order of ps, building up the significant vibrational state populations needed for nonlinear Raman favors intense short-pulse excitation, which has not typically been used for SERS, nor has the theory for this regime been derived. More recently, pioneering coherent-anti-Stokes Raman scattering (CARS) has been applied to single plasmonic dimers [34], highlighting weak signals and rapid damage as noted below, which make power-dependent measurements to probe optomechanical models challenging. Here, we study intensity-dependent SERS from single gold nanoparticles under pulsed and continuous wave (CW) illumination.

\section{EXPERIMENTAL SETUP AND RESULTS}

A nanoparticle-on-mirror (NPoM) [35,36] configuration is adopted [Fig. 1(c), inset] to provide robust reproducible plasmon enhancements on self-assembled molecular monolayers (SAMs) sandwiched inside the gap. The extreme field localization for small 1-2-nm gaps between the nanoparticle and metal film, as well as the oriented molecules, makes this geometry a perfect platform to closely investigate molecular dynamics and local morphological reconfiguration within the nanocavity under laser illumination. The Stokes (S-SERS) emission is obtained for ps-pulsed as well as $\mathrm{CW}$ lasers with increasing incident powers, revealing three different regimes. Dark-field scattering and SERS measurements show that at higher powers, gold atoms start to irreversibly move around and at the same time new molecular transitions are observed. Our data imply movement of surface $\mathrm{Au}$ atoms and surface-bound molecules, leading ultimately to the breaking of molecular bonds [Fig. 1(a)].

Samples are prepared by drop casting $80-\mathrm{nm}$ gold nanoparticles onto a molecular-coated gold film (Appendix A). A biphenyl-4-thiol (BPT) SAM is used as the spacer, with well-defined thickness $d=1.3 \pm 0.1 \mathrm{~nm}$ confirmed by ellipsometry. By using a low nanoparticle density on the sample, measurements can be performed on individual NPoMs, initially using dark-field scattering spectroscopy [Fig. 1(d) black line]. Typical spectra show two plasmonic modes: the characteristic gold transverse plasmon resonance $T(\lambda \sim 530 \mathrm{~nm})$ and a longitudinal bonding dimer plasmon (BDP) mode $L$ in the near-infrared $(\lambda \sim 720 \mathrm{~nm})$, due to dipolar coupling between the nanoparticle and its image charges in the metal film. After optical characterization of each NPoM, power-controlled Raman measurements are performed at a fixed $\lambda=633 \mathrm{~nm}$ excitation wavelength, switching between a pulsed laser source and an equivalent CW laser [Fig. 1(b)]. In the former case, we produce 1-ps pulses by spectrally filtering the 200 -fs pulses from an optical parametric oscillator with an acousto-optic programmable dispersive filter (Appendix A) to suitably improve the spectral resolution of our system $(<1 \mathrm{~nm})$. A variable neutral density filter adjusts the incident laser intensity, with average power $\langle I\rangle=\left(1 / T_{r}\right) \int_{0}^{T_{r}} I(t) d t$ for pulse trains with repetition rate $T_{r}=13 \mathrm{~ns}$ (much longer than the phonon decay time).

The strongly localized electromagnetic field enhancement $\left(>200 E_{0}\right.$ ) resulting from the plasmonic coupling between the nanoparticle and the gold film [37,38] allows the detection of the vibrational signature of BPT molecules confined within the nanogap. The number of molecules in each hot spot contributing to the total Raman signal is estimated to be around 100 in this system [39]. Identical SERS signals are obtained from each individual investigated NPoM [Fig. 1(c)], always showing three dominant vibrational lines corresponding to the $C-H$ rocking mode $\left(1080 \mathrm{~cm}^{-1}\right)$ and to in-plane stretching of the benzene rings $\left(1256 \mathrm{~cm}^{-1}\right.$ and $\left.1585 \mathrm{~cm}^{-1}\right)$. Using pulsed excitation gives the same SERS spectrum for BPT [broadened by the ps pulse, Fig. 1(e), as studied in detail in Ref. [28]], from which we extract the total integrated SERS for each peak, 
as well as the SERS background (around $2000 \mathrm{~cm}^{-1}$ ), which arises from the inelastic scattering of electrons in the metal. The power dependence of these two components [Fig. 1(f)] shows that the molecular Raman is nonlinear while the electronic Raman is linear. Explaining this observation is the main focus of this paper.

\section{MODEL AND DISCUSSION}

A nonlinear evolution of the Stokes Raman signal for specific pump detuning is a strong indication that optomechanical interactions between the plasmons of the cavity and the vibrations of the molecules are occurring in our system $[19,20,40]$. We describe the theoretical basis for understanding Raman signals within pulsed molecular optomechanics in the following. The coupling between confined plasmons at cavity frequency $\omega_{c}$ (with bosonic annihilation and creation operators $\hat{a}, \hat{a}^{\dagger}$ ) and phonons of frequency $\omega_{m}$ (operators $\hat{b}, \hat{b}^{\dagger}$ ), driven by an external laser at $\omega_{l}$ of field strength $A(t)$, is governed by the nonlinear optomechanical Hamiltonian $[19,20]$

$$
\begin{aligned}
\hat{H}= & \hbar \omega_{c} \hat{a}^{\dagger} \hat{a}+i \hbar A(t)\left(\hat{a}^{\dagger} e^{-i \omega_{l} t}-\hat{a} e^{i \omega_{l} t}\right) \\
& +\hbar \omega_{m} \hat{b}^{\dagger} \hat{b}-\hbar g_{0} \hat{a}^{\dagger} \hat{a}\left(\hat{b}+\hat{b}^{\dagger}\right)
\end{aligned}
$$

This description is completed by the inclusion of the spontaneous decay of phonons with rate $\gamma_{m}$ and plasmons with decay rate $\kappa$ (see Appendix I for details). In the weak single-photon coupling regime (when $\left.g_{0} \ll \omega_{m}, \kappa\right)$ the above Hamiltonian can be linearized, and the optical degree of freedom can be traced out $[19,20,40]$. As a result, a convenient description of the dynamics of the vibrational degree of freedom can be formulated, in which the rate of creation and annihilation of phonons is modified by the presence of the cavity, acting now as a reservoir of excitations for the vibrations. Throughout this process, the cavity is assumed to be in a coherent state, and the phonons are in a thermal state, with negligible coherent amplitude and dominant incoherent population (see Appendix I for details).

We consider the response of the plasmonic cavity to either $\mathrm{CW}$ or pulsed illumination. In the former case, the cavity is assumed to be in a steady coherent state, defined by the optical amplitude $\alpha=A /\left[i\left(\omega_{c}-\omega_{1}\right)+\kappa / 2\right]$. On the other hand, the temporal length of the incident pulse used in this study falls in between the characteristic evolution times of the cavity plasmons (few fs) and phonons (few ps). The amplitude of the coherent state of plasmons $\alpha(t)$ thus follows the temporal shape of incident pulse $I(t) \propto|A(t)|^{2}$ almost instantaneously. Indeed, we verify through numerical integration of the master equation that when illuminated by a 1-ps Gaussian pulse, the cavity behaves as if illuminated by a $\mathrm{CW}$ source with instantaneous pumping rate $A(t)$, and after approximately $20 \mathrm{fs}$, it settles into a coherent state with amplitude $\alpha(t)=A(t) /\left[i\left(\omega_{c}-\omega_{l}\right)+\kappa / 2\right]$.

A similar analysis suggests that we cannot assume that the vibrations reach a steady state when the system is illuminated by a 1-ps pulse. The solution to the optomechanical system dynamics adopted here is thus formulated in terms of the dynamics of the mechanical degree of freedom, assisted by the optical cavity, which has settled into a steady state. In particular, the dynamics of the population of phonons is described by the rate equation $[40,41]$ :

$\frac{d}{d t} n_{\text {phon }}=-n_{\text {phon }}\left(\gamma_{m}+\Gamma_{-}\right)+\left(n_{\text {phon }}+1\right) \Gamma_{+}+\gamma_{m} n_{t h}$.

Here $n_{\mathrm{th}}$ is the thermal population of phonons due to the finite temperature of the environment $T=300 \mathrm{~K}$, varying from $n_{\text {th }}=10^{-4}$ to 0.03 for the typical vibrational frequencies of the BPT molecule we observe. The role of the cavity is captured by the Stokes $\left(\Gamma_{+}\right)$and anti-Stokes $\left(\Gamma_{-}\right)$cavityassisted transitions rates. As each Stokes transition adds a phonon to the molecule and each anti-Stokes transition removes one, the optomechanical damping defined as $\Gamma_{\mathrm{opt}}=\Gamma_{-}-\Gamma_{+}$describes how a plasmonic cavity can either enhance (for positive $\Gamma_{\text {opt }}$ ) or suppress (for negative $\Gamma_{\text {opt }}$ ) the effective decay rate $\Gamma_{\text {eff }}=\gamma_{m}+\Gamma_{\text {opt }}$ of phonons. While this behavior is reminiscent of a harmonic oscillator resonantly coupled to a structured bath of excitations, here the transition rates $\Gamma_{ \pm}$exhibit a linear dependence on the plasmon population $n_{\mathrm{pl}}=|\alpha(t)|^{2}$, and consequently, on the pulse temporal evolution.

Considering first the situation of CW illumination, where the Stokes and anti-Stokes transition rates $\Gamma_{+}$and $\Gamma_{-}$are time independent, the steady-state phonon population is found to be

$$
n_{\text {phon }}=\frac{\gamma_{m}}{\gamma_{m}+\Gamma_{\mathrm{opt}}} n_{\mathrm{th}}+\frac{\Gamma_{+}}{\gamma_{m}+\Gamma_{\mathrm{opt}}}
$$

For negative optomechanical damping $\Gamma_{\text {opt }}$, this solution exhibits a threshold behavior when the cavity amplitude becomes large enough for the effective phonon damping $\Gamma_{\text {eff }}$ to vanish. We demonstrate this behavior in Fig. 2(a), where we plot the phonon population and effective optomechanical damping as a function of the intensity of the CW illumination $I \propto|A|^{2}$, with solid and dashed black lines, respectively. These plots are calculated for the $1585 \mathrm{~cm}^{-1}$ vibrational mode, with system parameters fitted to the experimental results (see below). For the lowest laser powers, the thermal population of phonons $n_{\text {th }} \approx 5 \times 10^{-4}$ dominates. Since in this unpumped regime the optomechanical damping $\Gamma_{\mathrm{opt}} \propto I$ is negligible, the population of phonons in Fig. 2(a) remains constant. For larger laser powers between $10^{1}$ and $10^{3} \mu \mathrm{W} / \mu \mathrm{m}^{2}$, the optomechanical pumping remains very small $\left(-\Gamma_{\mathrm{opt}} \ll \gamma_{m}\right)$ so that the normalized effective decay rate $\Gamma_{\text {eff }} / \gamma_{m}=\left(\Gamma_{\text {opt }}+\gamma_{m}\right) / \gamma_{m}$ 

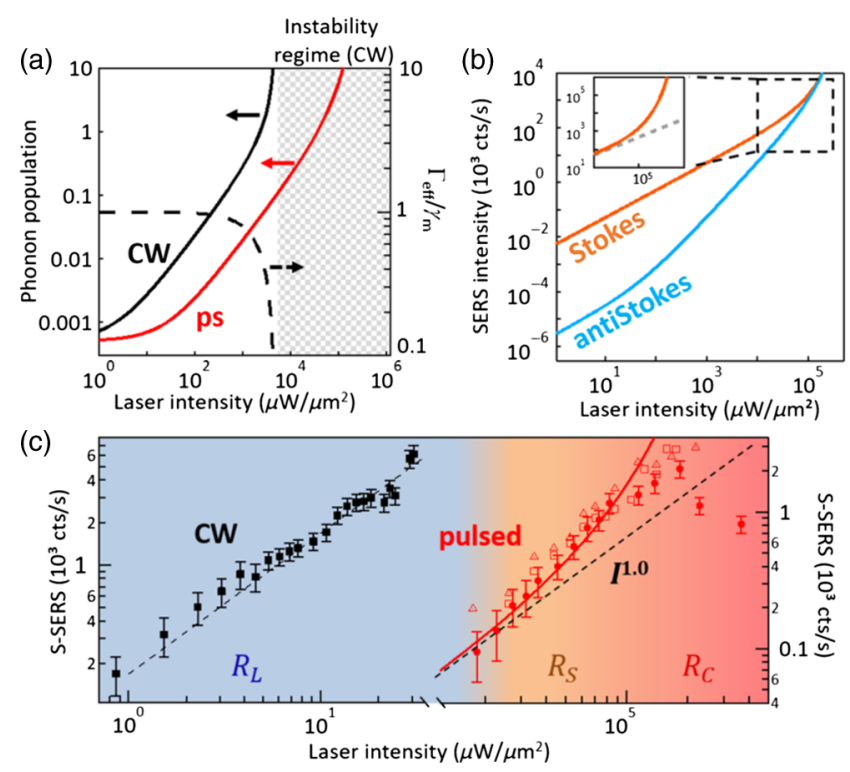

FIG. 2. Runaway molecular vibrations. (a) Comparison of the nonlinear buildup of incoherent phonon population (solid lines) for CW (black line) or pulsed (red line) illumination. In the latter case with 1-ps Gaussian pulses, the peak laser intensity and phonon population are used. Only for CW illumination does a clear threshold arise from the vanishing effective phonon decay rate (dashed line, normalized to the free-space phonon decay rate), interpreted as the phonon lasing (phasing) or instability regime. (b) Stokes (orange line) and anti-Stokes (blue line) dependence on the peak intensity of the pulsed illumination. Zoomed region (inset) highlights the superlinear predicted timeintegrated Stokes emission (linear shown dashed). (c) StokesSERS intensity of $1585 \mathrm{~cm}^{-1}$ mode for a single NPoM vs peak laser intensity for (red circles) ps-pulsed and (black squares) CW excitation. Pulsed measurements on two additional particles are also shown (red triangles, squares). Linear $\left(R_{L}\right)$, superlinear $\left(R_{S}\right)$, and driven-chemical $\left(R_{C}\right)$ regimes are highlighted in blue, orange, and red. The black dashed lines show a linear response; the red line shows Stokes emission calculated from the model in (b).

remains unity [black dashed line, Fig. 2(a)]. However, the vibrational pumping mechanism starts to dominate over the thermal population, yielding a phonon population linearly dependent on $I$. Finally, near the threshold, the magnitude of optomechanical damping $\Gamma_{\text {opt }}$ becomes comparable to $\gamma_{m}$; consequently, the effective phonon damping decreases rapidly and the phonon population reaches a regime of nonlinear, asymptotic growth. We note that in SERS, the rapid increase of the phonon population might then be suppressed by the onset of anharmonicity of the electronic potential of the molecule.

For pulsed illumination (with $I$ redefined as the illumination peak intensity), the intensity dependence of the phonon population is somewhat different. As shown in Fig. 2(a) (red line), the vibrational pumping of phonons becomes less efficient than under $\mathrm{CW}$ illumination, and the system does not exhibit such a clear threshold behavior.
Instead, the phonon population becomes superlinearly dependent on $I$ for significantly larger peak laser powers (around $10^{5} \mu \mathrm{W} / \mu \mathrm{m}^{2}$ ), and asymptotically converges to an exponential growth determined by the parameters of illumination.

While the dynamics of vibrations is very different for $\mathrm{CW}$ and pulsed illumination, in both cases the Stokes and anti-Stokes emission intensities are determined by the product of the laser intensity and phonon population as

$$
S_{S} \propto I\left(1+n_{\mathrm{phon}}\right), \quad S_{\mathrm{AS}} \propto I n_{\mathrm{phon}}
$$

This emission is integrated over the duty cycle. In Fig. 2(b), with solid lines, we plot the Stokes and antiStokes intensities for pulsed illumination, as a function of the peak laser intensity. In the thermal regime (below $10^{2} \mu \mathrm{W} / \mu \mathrm{m}^{2}$ ), emission from both the Stokes and antiStokes is linearly proportional to $I$. In the vibrational pumping regime (between $10^{2}$ and $10^{4} \mu \mathrm{W} / \mu \mathrm{m}^{2}$ ), $n_{\text {phon }} \propto I$, and the anti-Stokes emission has a quadratic dependence on $I$. Finally, a region of significant nonlinearity of Stokes emission, for laser intensities over $10^{4} \mu \mathrm{W} / \mu \mathrm{m}^{2}$, is magnified in the inset (dashed line shows linear dependence). We note that these dependencies of Stokes and anti-Stokes emission in Eq. (3) can also be derived from a purely classical theory, by solving the rate equation for the phonon population $[40,41]$.

Such predicted behaviors are seen in the experiment. Exciting single NPoMs with average intensities below $10 \mu \mathrm{W} / \mu \mathrm{m}^{2}\left(\sim 10^{13}\right.$ photons/s or, given the plasmon cavity lifetime of $20 \mathrm{fs}$ and small cross section, far less than one plasmon excited at any time), the dark-field scattering spectrum remains unmodified [Fig. 1(d), red line] and the SERS intensity increases linearly with laser average intensity [regime $R_{L}$ in Fig. 2(c), black squares]. This is characteristic of both the thermal and vibrational pumping regimes. Similar linear behavior is seen for pulsed excitation with peak intensities below $10^{4} \mu \mathrm{W} / \mu \mathrm{m}^{2}$ and is well reproduced by the low-power limit optomechanical theory of SERS [left-hand side of Fig. 2(b)]. In this regime, as expected, absolute S-SERS intensities are similar for $\mathrm{CW}$ and pulsed excitation of the same average power (Appendix B).

A complete change is seen for pulsed peak powers $>10^{4} \mu \mathrm{W} / \mu \mathrm{m}^{2}$ [Fig. 2(c)], with the Stokes SERS intensity now showing a clear superlinear increase [Fig. 2(c), superlinear regime $R_{S}$ ] before saturation sets in. This nonlinear behavior matches the molecular optomechanical model adapted to pulsed excitation, depicted with the red line (using phonon decay rates $\hbar \gamma_{m}=0.07 \mathrm{meV}$ and single-photon coupling parameter $\hbar g_{0}^{\text {fit }}=6 \mathrm{meV}$, consistent with the data obtained from dark-field spectroscopy of the cavity and Raman spectroscopy of the molecules). Previously we showed that anti-Stokes emission (AS-SERS) is indeed nonlinear in the vibrational pumping regime for small 
populations [8], consistent with the established characteristic of the vibrational pumping regime, but with only a linear SSERS signal. Here we show that at much larger peak laser powers, the Stokes signal also becomes superlinear as the phonon populations become nonlinear. This is a clear signature of the amplification of the phonon population in molecules due to optomechanical backaction coupling to the plasmonic mode, revealing the stimulated precursor to the parametric instability (Appendix I). This latter regime is, however, not accessible with stronger CW pumping, as we discuss later.

The evolution of SERS intensity in single NPoMs with increasing laser powers is tracked for the three fundamental Raman peaks of BPT in the case of ps-pulse excitation (Fig. 3). It is evident that the three modes share a similar nonlinear power dependence, with higher power onsets of nonlinear emission for lower frequency modes, but similar power thresholds for saturation (results on different NPoMs give thresholds that vary $\pm 50 \%$ ).

Since many molecules $\left(N_{m} \sim 100\right)$ are present in the NPoM cavity, it is important to consider how this ensemble couples to the plasmonic mode. The two extreme cases are (a) that all molecules vibrate collectively or (b) that each molecule is coupled individually to the plasmonic mode and no coherence arises between the vibrations of molecules. In the latter case, the coupling strength is simply given by the single-molecule coupling $g_{0}$, with all phonons localized to a specific molecule. On the other hand, for the collective vibrations of case (a), the overall coupling strength is expected to be $g_{N}=g_{0} \sqrt{N_{m}}$, with the phonons delocalized across all molecules. In more realistic systems, however, the molecules will be dispersed inside the cavity and exhibit a distribution of single-plasmon, singlemolecule coupling parameters $g_{0}$.

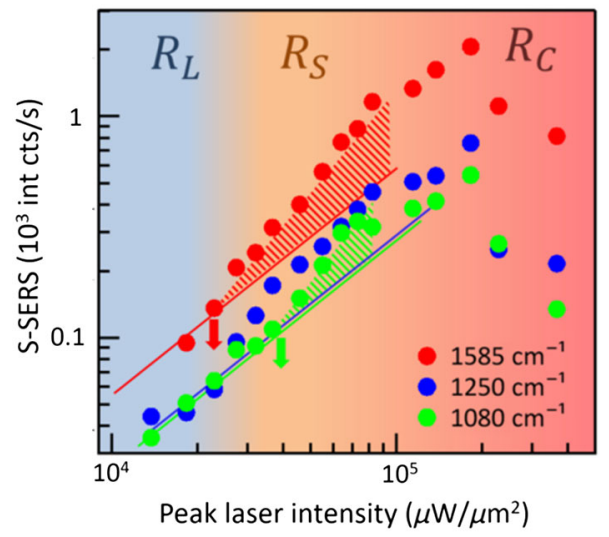

FIG. 3. Regimes for different molecular vibrations. SERS intensity of the $1585 \mathrm{~cm}^{-1}, 1250 \mathrm{~cm}^{-1}$, and $1080 \mathrm{~cm}^{-1}$ modes for BPT molecules forming the plasmonic spacer, as a function of the incident pulsed laser power: linear $\left(R_{L}\right)$, superlinear $\left(R_{S}\right)$, and driven chemical $\left(R_{C}\right)$ regimes are highlighted in blue, orange, and red, respectively. Lines show linear fits to the $R_{L}$ regime; arrows show thresholds.
The different coupling strengths in cases (a) and (b) correspond to different onsets of the nonlinear Stokes emission $[19,20]$. From the optomechanical parameters here (given in Appendix I), we estimate the single-plasmon optomechanical coupling as $\hbar g_{0} \sim 0.38 \mathrm{meV}$, and hence $\hbar g_{N=100} \sim 4 \mathrm{meV}$. The theoretical fit to the experimental results shown in Fig. 2(c) was calculated using $\hbar g_{N}=6 \mathrm{meV}=\hbar g_{0}^{\mathrm{fit}}$, which suggests that a strong collective behavior is indeed observed for the first time. For $\mathrm{CW}$ illumination, the nonlinear threshold is determined directly by the ratio $\left(g_{0}\right)^{2} / \gamma_{m}$; however, under pulsed illumination, this onset of superlinear Stokes emission becomes largely independent of the free-space phonon decay rate $\gamma_{m}$ for typical values of this parameter. This is because the phonon population does not reach equilibrium during the pulsed illumination, and its buildup is thus governed predominantly by the coupling parameter and illumination intensity, instead of the decay of the phonon population. We discuss this effect in more detail in Appendix J. We emphasize that in this power regime all changes in experimental SERS spectra are still completely reversible, and neither spectral shifts of the main Raman modes nor any relative change in their intensities are observed. A similar dependence is seen for all NPoMs.

In comparison to our previous experiments [8], in which we used cryogenic temperatures to stabilize "picocavities" based around single Au atom projections within the facets of the nanogap, here we remain in the nanocavity limit at room temperature, with optical fields delocalized laterally $\sim 5 \mathrm{~nm}$ and probing many molecules simultaneously. This is because we work at room temperature, and we avoid such high powers that move the $\mathrm{Au}$ atoms around (which is checked by dark-field scattering before and after each experiment). At room temperature, any picocavities are typically highly unstable, existing for much shorter times than the integration times here. This is confirmed from successively taken SERS spectra that show no fluctuations in vibrational peak positions, as expected for the 100 molecules probed, rather than the fluctuating energies seen for single molecules in picocavities [8]. It is very evident whenever a picocavity appears, showing sharp additional Raman lines that exhibit Stokes:anti-Stokes ratios close to unity, and we thus avoid these [42]. Given the inverse scaling of the optomechanical coupling $g_{0}$ on the plasmon cavity volume, we thus expect its hundredfold decrease from the picocavity to nanocavity regimes, as is indeed observed above (from $40 \mathrm{meV}$ in picocavities to $\hbar g_{0} \sim$ $0.4 \mathrm{meV}$ in these nanocavities).

To access the parametric instability the power is further increased, but for peak laser intensities $>10^{6} \mu \mathrm{W} / \mu \mathrm{m}^{2}$ (equivalent to average powers $>10 \mu \mathrm{W} / \mu \mathrm{m}^{2}$ ), the signal decreases irreversibly. As we discuss below, this is likely a result of chemical changes of the molecules attached to the gold and reconfiguration of the nanoparticle 
shape [Fig. 2(c), chemical regime $R_{C}$ ] [8]. We also stress that the ever-present SERS backgrounds (here comparable in strength to the SERS peaks, Appendix F) remain always in the linear regime, for both pulsed and CW excitation, confirming their separate origin to the molecular vibrational peaks [43].

To isolate the effects of pulsed excitation, we also take power dependence data on the same samples with $\mathrm{CW}$ excitation at the same wavelength. As observed in previous work $[44,45]$, at higher intensities irreversible behaviors appear (see Appendix E). The first effect is the appearance of sharp extra lines alongside the three main vibrational modes of BPT. Unlike the reversible pulsed nonlinear $R_{S}$ regime, these new modes, which are not found in the solution Raman spectra, are instead both spectrally and temporally unstable, appearing and evolving very rapidly $(<1 \mathrm{~s})$. This indicates more dramatic conformation changes involving dynamic bending, flexing, or partial bond breaking of the molecules (see Appendix H). Comparable changes are also seen in TERS [46], though driven by current instead of light.

Simultaneously with these changes in the vibrational spectra, the scattering spectra are observed to evolve, with $>50 \mathrm{~nm}$ redshifts [Fig. 4(a), black points] and decreasing amplitude of the plasmonic coupled mode. These spectral changes have been previously identified with movement of the metal atoms around the nanoparticle,

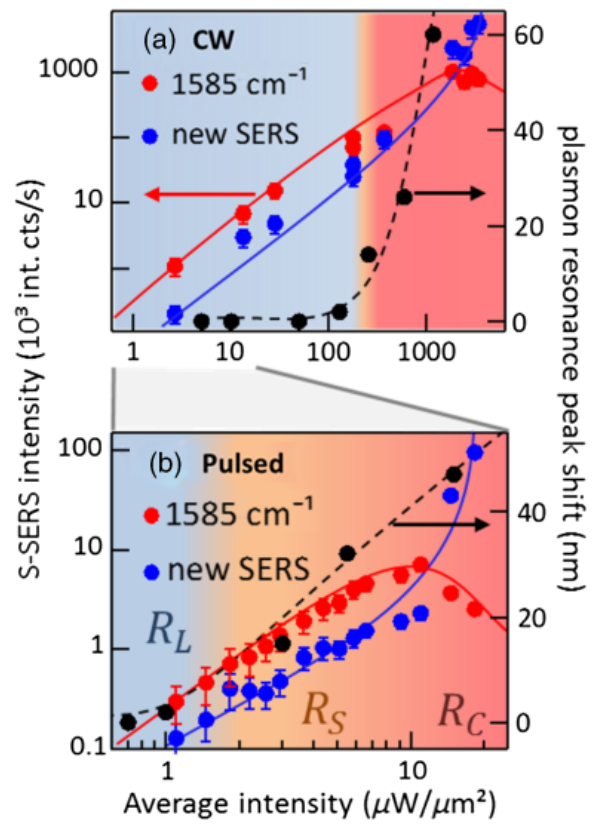

FIG. 4. Driven photochemical bond breaking. (a)-(b) StokesSERS intensities (left axis) for persistent sharp $1585 \mathrm{~cm}^{-1}$ mode (red points) and new SERS peaks (integrated, blue points) from a single NPoM for increasing average laser intensity $I$, with (a) CW and (b) pulsed excitation. The right axis (black points) shows the corresponding shifts of the coupled plasmon mode in simultaneous dark-field scattering spectra. Lines are guides for the eye. increasing the width of the bottom facet in contact with the molecules [37,47]. Diffusion of Au atoms was also suggested in [44], though the mechanism suggested there cannot apply here as our NPoM geometry constrains the molecular layer. Recent experiments show that thiol-SAM/ gold interfaces typically reconstruct via $S-\mathrm{Au}-S$ staples that pull atoms from the surface $[48,49]$, thus mixing molecular and metal atom dynamics. Similar effects for both pulsed and CW excitation are seen in the Raman and scattering, except that their threshold $I_{C}$ is at 100-fold lower average power for the pulsed case compared to the CW case [Fig. 4(b)]. This suggests that instantaneous phonon occupations (which are larger when pulsed excitation is faster than phonon decay than for CW excitation) are more important than any equilibrium heating of the system. In situ measurements of the temperatures of metal and molecules show mild $>10{ }^{\circ} \mathrm{C}$ temperature rises above the same threshold at $I_{C}$ (Appendix K), but these temperature rises are not capable of producing the Stokes nonlinearity seen above (Appendix L). We note that broadband $\mathrm{CW}$ photocatalysis of ethylene epoxidation for large ensembles of plasmonic gaps also shows superlinear nonlinearities in its efficiency at high powers $>10^{5} \mu \mathrm{W} / \mu \mathrm{m}^{2}$ [50], tentatively assigned to multiexcitation that increases occupation of higher vibrational states, and which aligns well with the mechanism outlined here.

Our measurements thus show passage from linear to chemical-transformation regimes under both pulsed and CW excitation of SERS, but with clear superlinear pumping of the phonons in the former case starting at dramatically lower average powers than in CW pumping. It thus appears that there may be a link between optomechanical interactions and molecule-cavity reconfiguration. We suggest that this can arise from cooperative movement of gold atoms and molecules seen as chemical changes in the fluctuating vibrational spectra, but verifying this is currently challenging. A number of other mechanisms can induce bond breaking, such as hot-electron injection [51-54]. Indeed, the threshold for this is expected to be lower for pulsed compared to $\mathrm{CW}$ excitation, and perhaps one way to discriminate between optomechanical vs hotelectron-driven chemistry is through detailed dependence on the pumping wavelength.

Further progress toward phonon lasing (beyond the nonlinear precursor observed here) is prevented by such chemical changes. Vibrationally pumping the molecules to approach phonon lasing will start to excite high-lying vibrational states. Phonon populations with $\bar{n}=0.4$ (reached here for the modeled data in Fig. 2) have a $1 \%$ chance of occupying the $n=3$ vibrational state. Since this is closer in energy to the potential barrier for bond breaking, the process can be switched on [Fig. 1(a)]. It thus appears that specially designed robust molecules are required for optomechanically driven phonon lasing, which might survive the vibrational cascade. 


\section{CONCLUSIONS}

In summary, we show that ultratight confinement of light in plasmonic cavities allows access to reversible optomechanical driving, which shows the distinct superlinear power dependence of the phonon population that is the precursor to the molecular parametric instability. Picosecond pulses that excite phonons faster than they decay are needed to pump the vibrational population sufficiently to observe this. We derive the optomechanical theory for this interaction, and we show that it has different behaviors in $\mathrm{CW}$ and picosecond pumping regimes. We find that our model can account for the nonlinear dependence of the SERS emission, and that it suggests that $\sim 100$ molecules act together optomechanically in their interaction with the tightly confined plasmons. At larger pumping rates, however, permanent molecule changes are induced that terminate this phonon amplification process. We emphasize that very similar effects are also observed when we use silver nanostructures, nanoparticles of different sizes, or different thiol molecules. Our study highlights an intimate connection between the optomechanical and chemical processes in such highly confined plasmonic cavities. Our results suggest new approaches for understanding the dynamics of optomechanics in nanoscale systems, for making robust SERS architectures for sensing (particularly at high intensities in order to study fast dynamics) as well as stimulating new studies of fundamental molecule-surface interactions, for instance, in catalysis. This work also suggests that new reaction chemistries might be accessed by driving specific phonons optomechanically when confining light tightly with molecules.

\section{ACKNOWLEDGMENTS}

We acknowledge financial support from EPSRC Grants No. EP/G060649/1, No. EP/K028510/1, No. EP/L027151/ 1, No. EP/G037221/1, No. EPSRC NanoDTC EP/L015978/ 1, and ERC Grant No. LINASS 320503. M. K. S. and J. A. acknowledge support from MINECO Project No. FIS201680174-P and the COST Action MP1403 "Nanoscale Quantum Optics" supported by COST (European Cooperation in Science and Technology). M. S. acknowledges support from the Australian Research Council (ARC) Discovery Project No. DP160101691. F. B. acknowledges support from the Winton Programme for the Physics of Sustainability. We thank Dr. Guolei Xiang (University of Cambridge, Chemistry Department) for support with the TGA measurements.

\section{APPENDIX A: EXPERIMENTAL METHODS}

\section{Sample preparation}

Gold substrates are prepared by evaporating 100-nm gold (Kurt J. Lesker Company, PVD 200) on a silicon (100) wafer
(Si-Mat, Germany) at a rate of $1 \AA / s$. To obtain atomically smooth films, a standard template-stripping method is used: silicon substrates are glued to the freshly evaporated gold using an epoxy glue (EpoTek 377) [55] and the resulting gold-epoxy-silicon sandwich is peeled off the silicon wafer. Self-assembled monolayers of biphenyl-4-thiols (SigmaAldrich, 97\%) are formed by submerging the freshly template-stripped substrates into a 1-mM solution in water-free ethanol (Sigma-Aldrich, reagent grade, anhydrous) for $24 \mathrm{~h}$ (for the other SAM layers, see Ref. [39]). The samples are subsequently thoroughly rinsed with ethanol and blown dry. 80-nm gold nanoparticles (BBI solutions, UK) are deposited by drop casting from the as-received solution. The deposition time is adjusted in order to obtain the desired nanoparticle coverage. The samples are rinsed with milliQ water in order to remove any salt residues.

\section{Dark-field spectroscopy}

Dark-field images are measured on a custom Olympus GX51 inverted microscope. A focused halogen lamp is used for illumination. The scattered light is collected through a $100 \times$ dark-field objective (LMPLFLN-BD, $\mathrm{NA}=0.8)$ and analyzed with a fiber-coupled $(50-\mu \mathrm{m}$ optical fiber) Ocean Optics QE65000 cooled spectrometer. A standard diffuser is used as a reference to normalize the white light scattering.

\section{Power-dependent SERS measurements}

SERS experiments are performed on the same modified Olympus GX51 inverted microscope used for darkfield spectroscopy. The laser beam is focused down to approximately a $1-\mu \mathrm{m}$ spot size on the sample using a $100 \times$ objective $(\mathrm{NA}=0.8)$. Raman scattering is collected through the same objective and analyzed with a Shamrock SR-303i fully automated spectrometer coupled with an EMCCD camera cooled to $-85^{\circ} \mathrm{C}$. For the current experiments, we use a 600 -lines/mm 650-nm-blazed grating. Rayleigh scattering is filtered out with a replaceable set of three long pass (for Stokes measurements) or short pass (for anti-Stokes measurements) linear variable filters (DELTA). This spectral filtering system allows the detection of a minimum Raman shift of $400 \mathrm{~cm}^{-1}$ over the studied spectral range. The system is calibrated using a silicon substrate as a reference. We use a He-Ne 633-nm laser and a tunable ultrafast optical parametric oscillator (Spectra Physics Inspire) tuned to $633 \mathrm{~nm}$ for continuous and pulsed excitation, respectively. The parametric oscillator is pumped by a 200-fs Ti:sapphire oscillator (Spectra Physics MaiTai delivering 200-fs pulses, FWHM $10 \mathrm{~nm}$, at an $80-\mathrm{MHz}$ repetition rate). The monochromaticity of the output beam is reduced below 1-nm spectral bandwidth using an acousto-optic programmable dispersive broadband filter (AOPDF, Dazzler, Fastlite [56]). This filter uses interactions between polychromatic acoustic and optical waves within a 
birefringent crystal, is fully automated, and provides average output powers of several $\mathrm{mW}$ for the ps pulses.

\section{SERS time series}

SERS time series (integration time $1 \mathrm{~s}$ ) are recorded using a modified Olympus BX51 microscope equipped with a long-working-distance $100 \times(\mathrm{NA}=0.8)$ objective. Nanoparticles are identified by dark-field microscopy. To record the SERS spectra, a fully motorized homebuilt Raman spectrometer is coupled to the microscope. A spectrally filtered 632.8-nm He-Ne laser (maximum output $21 \mathrm{~mW}$ ) is used as the excitation source. The elastically scattered laser light is filtered with two Semrock 633-nm StopLine single-notch filters. The signal is coupled into an Andor Shamrock i303 spectrograph and Newton EMCCD. The laser is focused to a diffraction-limited spot on the sample, and the laser intensity is adjusted through a motorized ND filter wheel.

\section{APPENDIX B: LASER-POWER-DEPENDENT RAMAN STUDY OF SILICON}

The Raman signal from a silicon sample is measured for increasing laser powers. We use a continuous-wave $633-\mathrm{nm} \mathrm{He}-\mathrm{Ne}$ laser and repeat with a pulsed tunable optical parametric oscillator tuned to the same wavelength (Fig. 5). In both cases, the Raman signal increases linearly with laser power, and identical signal amplitudes are obtained. This linear dependence is preserved also in the "nonlinear" and "chemically driven" regions observed in the NPoM geometry at the same powers. This difference cannot be explained by any simply classical theory of heating (according to which a nonlinear increase should be observed for increasing laser powers in all cases), but only by considering the absence of a plasmonic cavity and strong optomechanical coupling in the bulk silicon case.

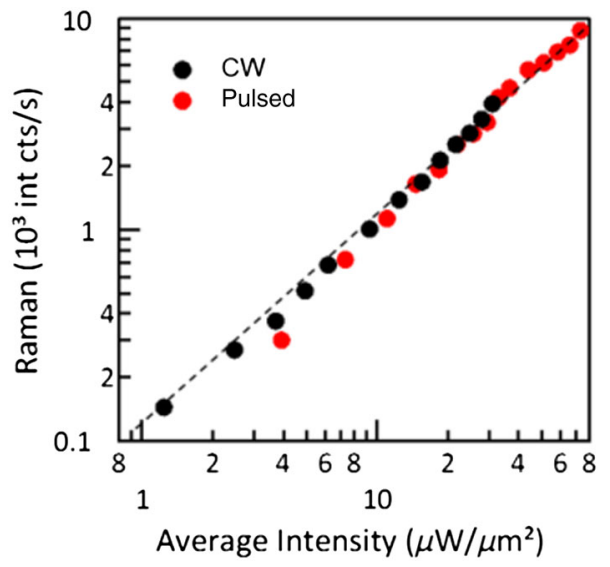

FIG. 5. Raman intensity of a silicon sample, as a function of excitation power for both $\mathrm{CW}$ and pulsed 633-nm excitations. Raman signal is plotted as a function of average laser intensity.

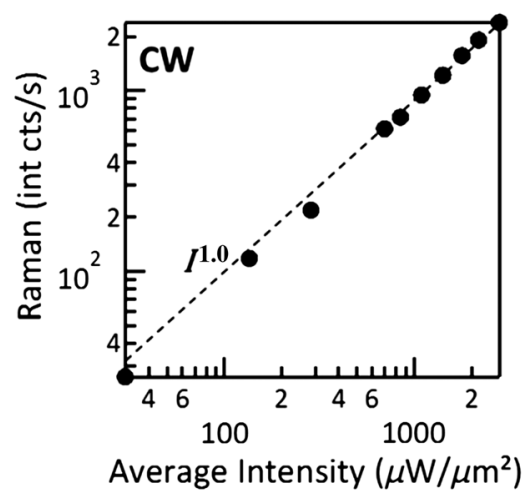

FIG. 6. Raman intensity of a bulk biphenyl-4-thiol sample, as a function of 633-nm CW excitation intensity.

\section{APPENDIX C: LASER-POWER-DEPENDENT RAMAN STUDY OF BULK BIPHENYL-4-THIOLS}

The vibrational Raman signal of bulk biphenyl-4-thiol powders is detected, and its intensity is plotted as a function of laser power. Because of the small Raman cross section of such samples, only CW excitation is used since it has higher spectral resolution. In this study, we can apply much higher powers (up to $2 \mathrm{~mW} / \mu \mathrm{m}^{2}$ ) as compared to the NPoM geometry, without observing any decrease in the Raman signal. The Raman increase follows a perfectly linear dependence on laser power up to the maximum power applied (Fig. 6).

\section{APPENDIX D: TGA MEASUREMENTS}

Thermogravimetric (TGA) analysis for a BPT sample is realized in an oxygen environment (Fig. 7). Evidence of a phase change is observed at $360 \mathrm{~K}$, while an endothermic melt happens at $390 \mathrm{~K}$. Evaporation, with consequent mass loss, starts around $450 \mathrm{~K}$.

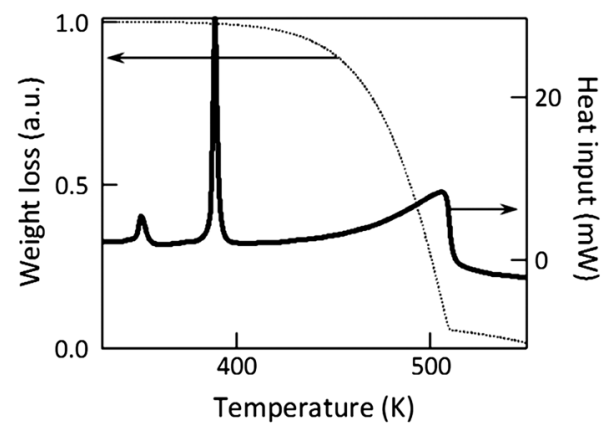

FIG. 7. TGA results for BPT powder. Measurements are realized in oxygen. 

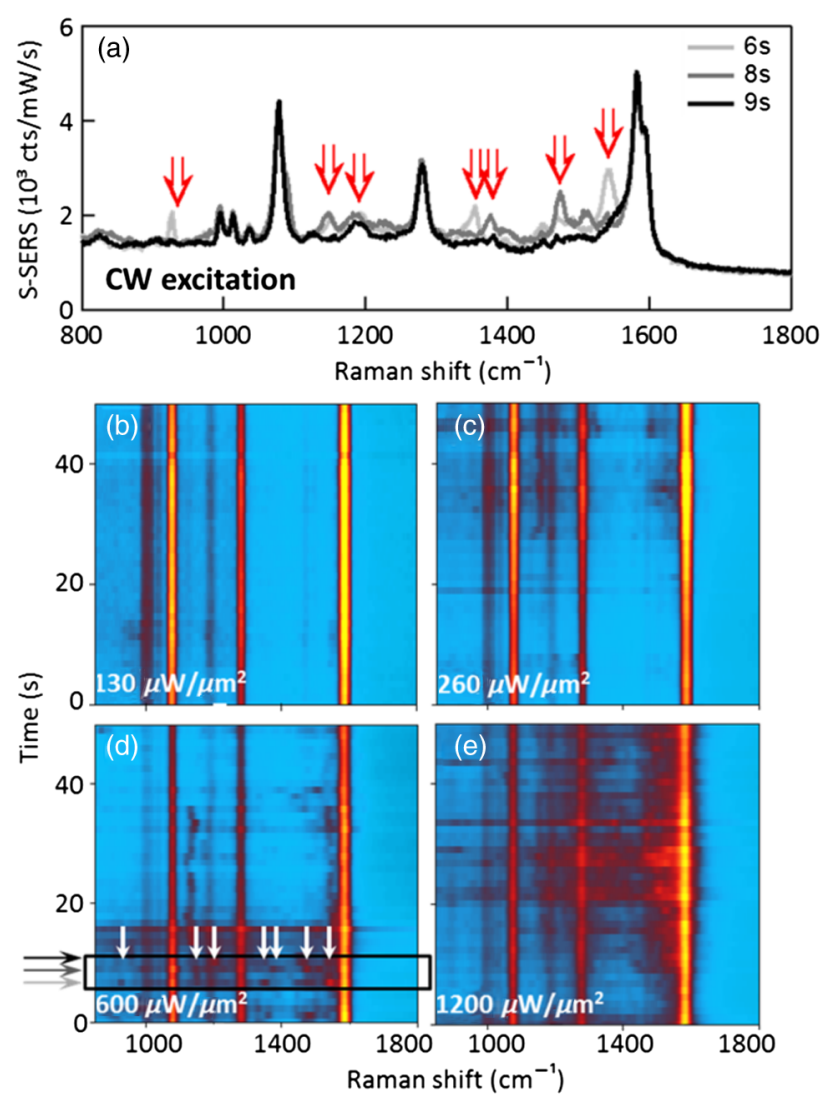

FIG. 8. Appearance of new Stokes-SERS lines in driven chemical regime. (a) S-SERS spectra at increasing times (3 s apart) from a single 80-nm gold nanoparticle-on-mirror showing evolution of new Raman modes (red arrows) for $I=$ $600 \mu \mathrm{W} / \mu \mathrm{m}^{2}$ CW 633-nm excitation alongside main BPT Raman modes. S-SERS time series for the same NPoM for increasing intensities (in $\mu \mathrm{W} / \mu \mathrm{m}^{2}$ ): (b) 130 , (c) 260 , (d) 600 , (e) 1200. The box in (d) shows spectra selected in (a).

\section{APPENDIX E: CW SERS TIME SERIES IN AIR AND VACUUM CONDITIONS}

The chemical transformation regime observed for pulsed excitation is seen also for CW pumping $>200 \mu \mathrm{W} / \mu \mathrm{m}^{2}$. We track the time evolution of S-SERS for individual gold NPoMs with BPT spacers in the CW case, identifying how new Raman lines appear sequentially [Fig. 8(a), red arrows on grey spectra]. Such behaviors have been seen previously in tightly confined plasmonic geometries $[44,45]$. The linear increase of the Raman transition signals with incident power already discussed at low intensities [Fig. 2(c)] is preserved up to $130 \mu \mathrm{W} / \mu \mathrm{m}^{2}$ [Fig. 8(b)]. For higher intensities, the first effect is the appearance of sharp extra lines alongside the three main vibrational modes of BPT [Figs. 8(a),(c)-(e)]. The reversible pulsed nonlinear $R_{S}$ regime for pulsed excitation does not apply to these new modes, which are instead both spectrally and temporally unstable. They appear and evolve very rapidly, often lasting only a few seconds [Figs. 8(a) and 8(d), vertical arrows].
These new vibrational frequencies are not found in solution Raman spectra and indicate more dramatic conformation changes involving dynamic bending, flexing, or partial bond breaking of the molecules [57].

Despite their intensity and spectral position changing dramatically for subsequent acquisitions (on the same particle), the intensity threshold for the chemical regime at which these lines start is very consistent $\left(I_{C} \approx\right.$ $300 \mu \mathrm{W} / \mu \mathrm{m}^{2}$ ) for different nanoparticles.

Repeating these measurements under vacuum gives identical results with identical rates. The SERS time series are realized on single NPoMs at low-pressure $(<1$ mbar) conditions, with increasing CW laser powers (Fig. 9). Despite the strong reduction of oxygen from the system (although surface bound oxygen might still be present), strong additional Raman lines rise at the same power threshold as recorded in ambient conditions, and at the same rate. This experiment suggests that the observed extra lines in the Raman spectrum are unlikely to be due to chemical products from oxidation reactions within the plasmonic nanogap.

The extra Raman lines arise at the same $I_{C}$ if a conducting BPDT molecular spacer is used, suggesting that the precise nature of the spacer has little influence on the final activation of the chemical regime. Gradually with further dosage, the extra lines start to fill the entire window between the initial Raman peaks, giving rise to a smooth background that must arise from the different configurations and chemical states of the 100 or so molecules in the plasmonic gap [Fig. 8(e)]. No such Raman effects are seen if bulk BPT powder is irradiated (Appendix C), indicating the importance of the plasmonic nanocavity in reaching the chemical regime. Previous work [44] has suggested the emergence of repeatable and correlated peaks, implicating a specific chemical reaction, in contrast to the appearance of lines in each case at different vibrational energies that we observe here.

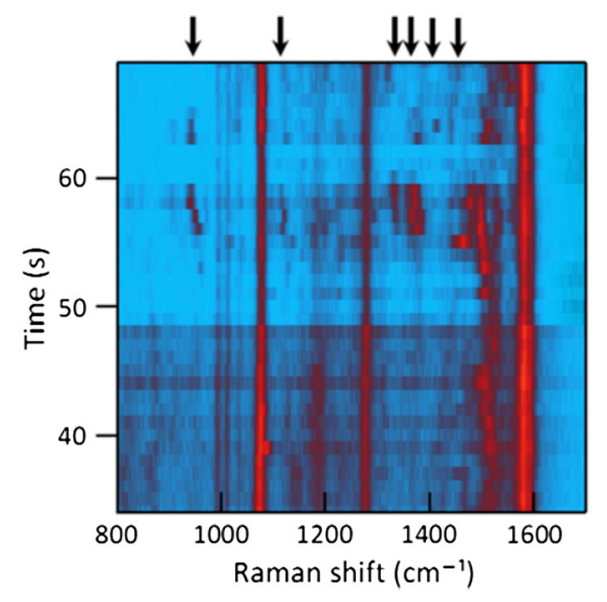

FIG. 9. SERS time series from a single 80-nm gold nanoparticle on mirror for $300 \mu \mathrm{W} / \mu \mathrm{m}^{2} \mathrm{CW}$ 633-nm laser excitation. Strong additional Raman lines (vertical arrows) arise and disappear in time, existing alongside the constant main BPT Raman modes. 


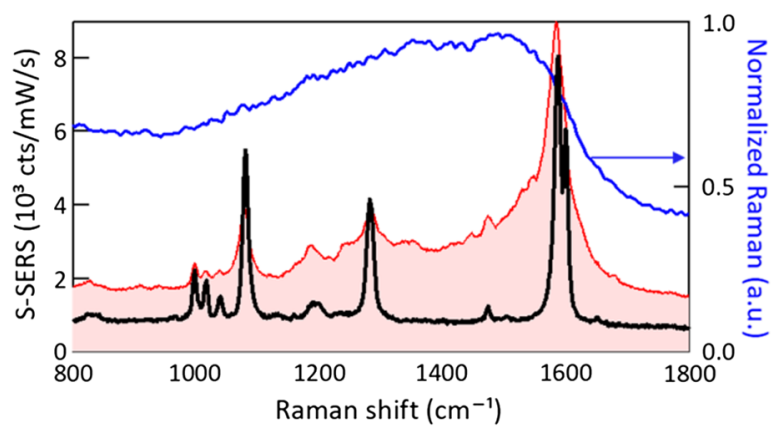

FIG. 10. SERS spectra from a single 80-nm gold nanoparticleon-mirror for a CW 633-nm low-power $I=260 \mu \mathrm{W} / \mu \mathrm{m}^{2}$ (black curve) and high-power $I=1200 \mu \mathrm{W} / \mu \mathrm{m}^{2}$ (red filled curve) excitation. Raman of a 1-nm-thick amorphous carbon layer on gold is also shown (blue line) as a comparison.

As new Raman lines appear, filling in the gaps between the vibrational resonances of the pristine molecular layer, the original SERS lines drop in intensity (red points in Fig. 4). It appears that the ordered molecules in the gap are thus gradually transformed into new species, with the delocalized $\pi$-orbitals successively being saturated. However, the SERS spectrum of every molecule is different, indicating their heterogeneous environments and bonding. This creates a final Raman continuum that resembles that of amorphous carbon (Fig. 10). In Appendix H, we provide density functional theory (DFT) calculations that show how different torsions, reconfigurations, and reactions can produce new Raman modes that sit in the spectral regions observed in the data (Figs. 8 and 9). Most surprising is the fact that the total integrated Raman emission increases through this process (see the blue points in Fig. 4), which suggests that the Raman cross sections of the molecules grow as their bonds reconfigure. These results thus suggest the many ways that permanent molecular changes give rise to the new modes observed.

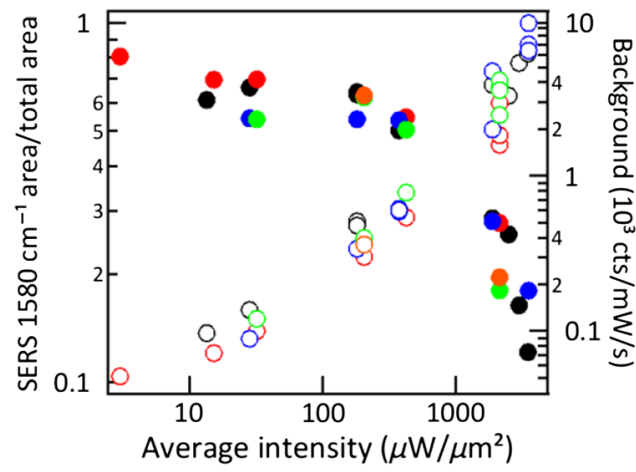

FIG. 11. Extracted relative intensity of the $1580-\mathrm{cm}^{-1}$ Raman mode detected from five individual NPoM (solid dots, left axis) and SERS background extracted at $2000 \mathrm{~cm}^{-1}$ (open dots, right axis), as a function of increasing $\mathrm{CW}$ laser power. Points of different color correspond to different nanoparticles.

\section{APPENDIX F: SERS POWER SERIES ON DIFFERENT PARTICLES (CW EXCITATION)}

The relative intensities of the $1580-\mathrm{cm}^{-1}$ Raman mode detected from several isolated nanoparticles on the mirror are extracted and plotted as a function of increasing average laser power for $\mathrm{CW}$ excitation (Fig. 11, left axis). All particles show a similar decrease in Raman intensity above a specific power threshold. The SERS background, on the other hand, always shows a linear increase with laser power (Fig. 11, right axis).

\section{APPENDIX G: SINGLE-PARTICLE SEM-OPTICAL MEASUREMENT CORRELATIONS}

The scattering spectrum of each measured gold nanoparticle is characterized through dark-field spectroscopy. A typical optical image of the sample is shown in Fig. 12(a). Each spot corresponds to an isolated particle on the gold substrate. The isolated nature of each particle is verified through scanning electron microscopy (SEM) measurements [Fig. 12(b)].

\section{APPENDIX H: DFT CALCULATIONS}

DFT calculations were performed to understand how modified molecules in the SAM can give rise to new vibrational lines in the regions observed. Structures were optimized and vibrational spectra were calculated using the Gaussian implementation of the B3LYP hybrid functional, augmented with a damped empirical dispersion term (GD3), using the LANL2DZ basis set (Fig. 13). The polarizable continuum model (CPCM) source field for water was used as a solvation model to simulate the possible presence of water in the system. The torsion range in the geometry was realized by fixing the dihedral angle between the two phenyl rings during geometry optimization. The proposed crosslinked structures are in part based on the cross-linking of BPT, forming carbon nanomembranes, known from literature [58].
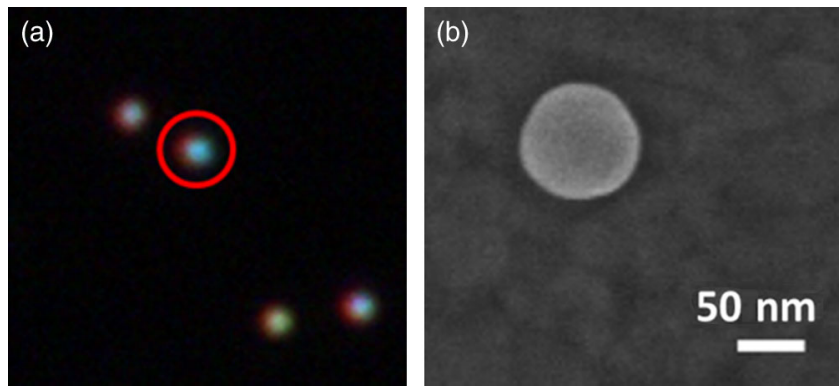

FIG. 12. (a) Dark-field optical image of a gold film with 80-nm gold nanoparticles deposited on top. (b) SEM image of the single particle highlighted in (a). 


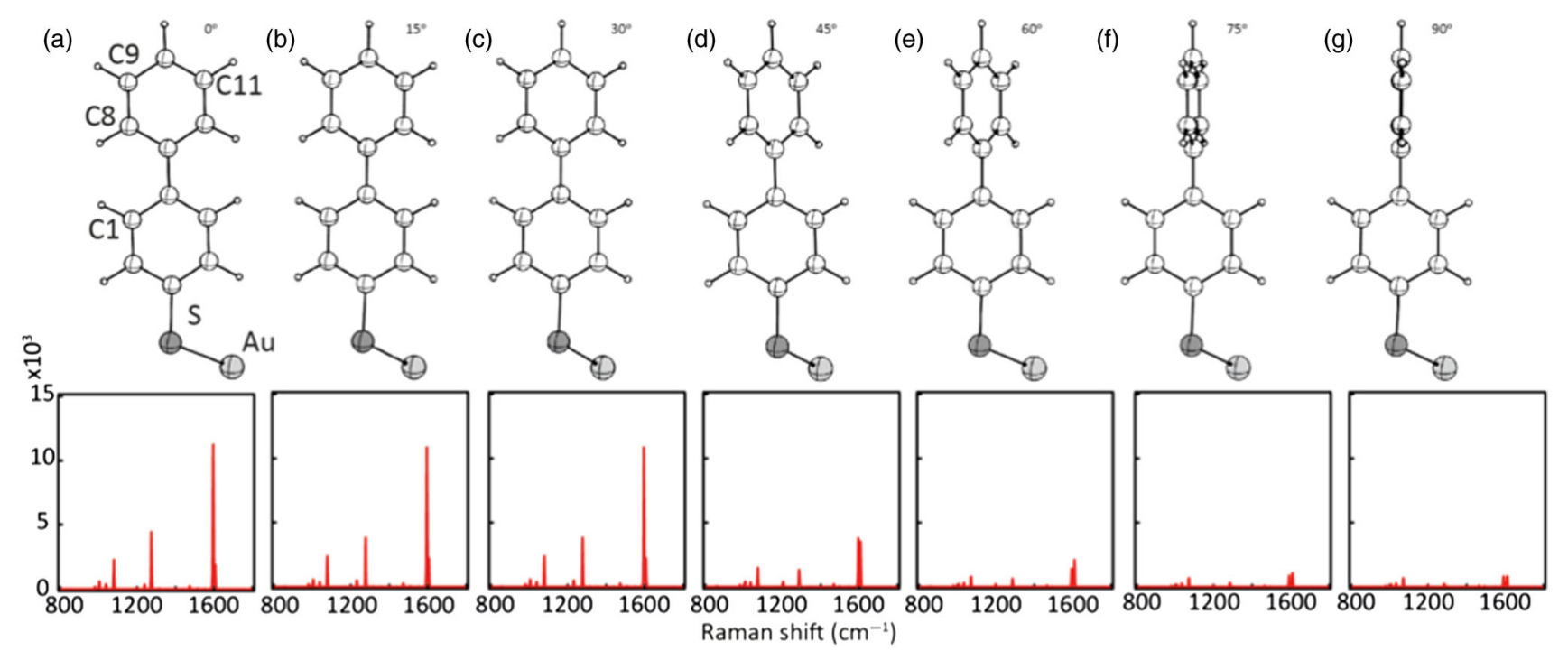

(h)
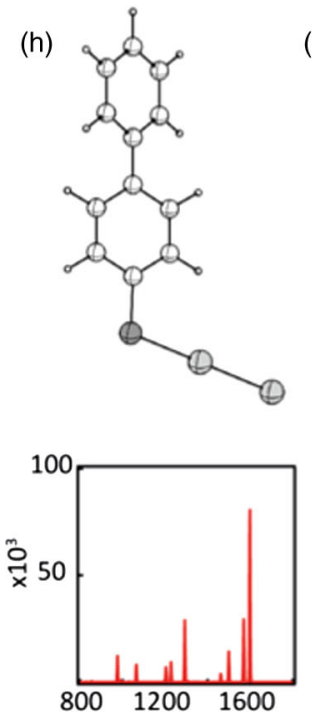
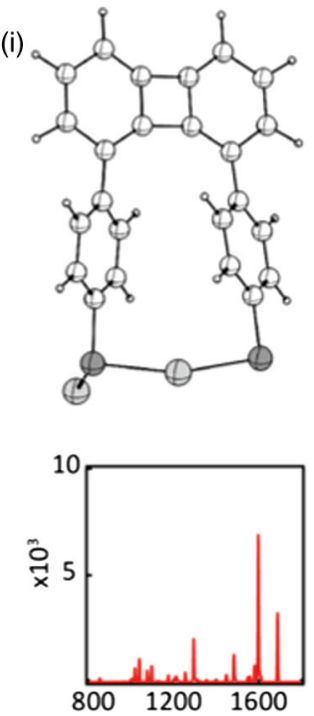
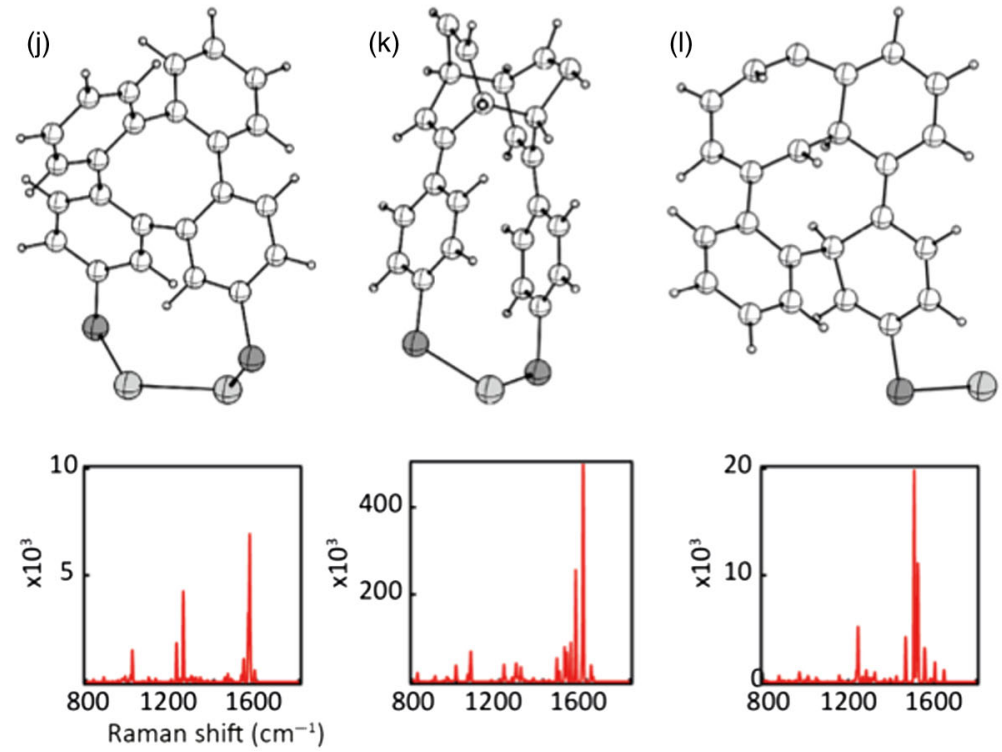

FIG. 13. Simulated 3D molecular structures for different BPT configurations and their corresponding Raman activity (below). (a) $-(\mathrm{g})$ Molecular torsion $\left(0-90^{\circ}\right)$ around central carbon linker for a single molecule. (h) Effect of the addition of an extra gold atom. (i)-(l) Possible cross-linked structures between neighboring BPT molecules after irradiation: (i) C8-C9 dimerization, (j) C1-C8 dimerization, (k) C8-C11 dimerization, and (1) C1-C8-C9 with ring-opening dimerization.

\section{APPENDIX I: MOLECULAR OPTOMECHANICS: THEORETICAL FRAMEWORK}

Recently, Roelli et al. [19], Schmidt et al. [20], and Benz et al. [8] suggested that the SERS process can be described in terms of a mechanical oscillator coupled to a cavity. In brief, Schmidt et al. considered the full optomechanical Hamiltonian given in Eq. (1) and provided a straightforward analytical solution in the case that the coupling strength of the oscillator to the cavity $g_{0}$ is much smaller than both the energy of the molecular vibration $\omega_{m}$ and the spectral width of the cavity $\kappa$ (which is the case in our system) $[8,20,40]$. We show here the equations required to reproduce Fig. 2 (main text) for CW as well as for pulsed illumination.
Within the theoretical framework of molecular optomechanics, the Stokes and anti-Stokes intensities $S_{S}$ and $S_{\mathrm{AS}}$ are given by

$$
\begin{gathered}
S_{S} \propto\left(\omega_{l}-\omega_{m}\right)^{4} \Gamma_{+}\left(n_{\mathrm{phon}}+1\right) \\
S_{\mathrm{AS}} \propto\left(\omega_{l}+\omega_{m}\right)^{4} \Gamma_{-} n_{\mathrm{phon}},
\end{gathered}
$$

where $n_{\text {phon }}$ is an incoherent phonon population (see discussion below), and parameters $\Gamma_{ \pm}=$ $g_{0}^{2} n_{\mathrm{pl}} \kappa\left[\left(\omega_{c}-\omega_{l} \pm \omega_{m}\right)^{2}+(\kappa / 2)^{2}\right]^{-1}$ describe the rates of Stokes and anti-Stokes transitions mediated by the plasmon in a single-cavity mode limit. The parameter $n_{\mathrm{pl}}$ describes 
the plasmon population in the nanocavity. To good approximation, if the coherent pumping $A$ is approximately constant on the time scale of the plasmon lifetime (i.e., for $\mathrm{CW}$ illumination or for 1-ps Gaussian pulses incident on a typical plasmonic cavity), this population is given by [40]

$$
n_{\mathrm{pl}}(t)=|\alpha(t)|^{2}=\frac{|A(t)|^{2}}{\Delta^{2}+(\kappa / 2)^{2}}
$$

with the laser detuning from the plasmonic cavity $\Delta=$ $\omega_{c}-\omega_{l}$ (with $\omega_{c}$ cavity resonance frequency and $\omega_{l}$ laser frequency), the spectral width of the cavity $\kappa$, so that the coherent pumping $A(t)$ drives the cavity into a coherent state with amplitude $\alpha(t)$. In the case of pulsed illumination, the Stokes and anti-Stokes transition rates $\Gamma_{ \pm}$are therefore implicitly time dependent through the evolution of $n_{\mathrm{pl}}(t)$, and follow closely the temporal shape of the pulse. We illustrate the effect of such a pulsed illumination on the dynamics of phonons in Appendix J.

The coherent pumping amplitude $A(t)$ can be calculated using the effective mode volume of the plasmonic cavity $V_{\text {eff }}$, the permittivity of the molecular layer $\epsilon_{g}$, the maximum near-field enhancement of the electric field in the plasmonic cavity $K$, and the laser power density $I$ [59]:

$$
A=\frac{\kappa}{2} \sqrt{\frac{\epsilon_{g} \epsilon_{0} V_{\mathrm{eff}}}{2 \hbar \omega_{c}}} K \sqrt{\frac{2 I}{\epsilon_{0} c}} .
$$

Here we use the following parameters for our experimental setup: laser wavelength $633 \mathrm{~nm}$, cavity resonance wavelength $720 \mathrm{~nm}$ (bonding-dimer plasmon mode), $\hbar \kappa=0.2 \mathrm{eV}$, permittivity of $\epsilon_{g}=2.10, V_{\text {eff }}=153 \mathrm{~nm}^{3}$, and $K=206$ (determined carefully from finite difference time-domain (FDTD) simulations at this wavelength).

Because of the spontaneous nature of the Stokes and anti-Stokes emission (albeit initiated by the absorption of a real cavity plasmon), the vibrations will be in a displaced thermal state, with a negligible coherent amplitude $\beta$ and a thermal-like incoherent population $n_{\text {phon }}$ evolving in time according to the rate equation given in Eq. (2) (note that $|\beta|^{2} \ll n_{\text {phon }}$; see [40] for a detailed discussion). The contribution to population due to the finite temperature of the environment is given by $n_{\text {th }}=\left[\exp \left(\hbar \omega_{m} /\left(k_{B} T\right)\right)-1\right]^{-1}$ (with $k_{B}$ the Boltzmann constant, and $T$ the absolute temperature). Under CW illumination, Eq. (2) can be solved to yield the steady-state phonon population given in Eq. (3), with the second term often expressed as

$$
\frac{\Gamma_{\mathrm{opt}}}{\gamma_{m}+\Gamma_{\mathrm{opt}}} n_{\mathrm{rad}}, \quad \text { where } n_{\mathrm{rad}}=\frac{\left(\Delta-\omega_{m}\right)^{2}+(\kappa / 2)^{2}}{4 \Delta \omega_{m}} .
$$

\section{Calculation of the coupling strength}

The coupling strength $g_{0}$ is determined both by the properties of the molecules and the properties of the cavity.
Assuming that the molecule is optimally positioned and oriented in the cavity, the maximum coupling parameter is defined as

$$
g_{0}=\frac{1}{2} \sqrt{\frac{\hbar R_{k}}{2 \omega_{m}}} \frac{\omega_{c}}{\epsilon_{0} \epsilon_{g} V_{\mathrm{eff}}}
$$

with Raman activity $R_{k}$, and the vacuum permittivity $\epsilon_{0}$ [20]. From DFT simulations, we find that the Raman activity of the mode used here $\left(1585 \mathrm{~cm}^{-1}\right)$ is $R_{k}=5085 \AA^{4} / \mathrm{amu}$. We note that this formula includes an additional factor of $1 / 2$ absent in previous formulations $[8,19,20]$ as we did not account there for the fact that the Raman dipole is not a permanent dipole, but is rather induced by the electric field of the plasmonic cavity. This calculation yields the single-plasmon optomechanical coupling $\hbar g_{0}=0.38 \mathrm{meV}$.

\section{Effects of collective coupling}

For a distribution of $N_{m}$ molecules coupled to the cavity, one can expect the formation of a superradiant phononic system with effective coupling given by $g_{0} \sqrt{N_{m}}$ [19]. Furthermore, the Raman emission linewidths should then become $\gamma_{m}+N \gamma_{\text {opt }}$. For $N_{m}=100$ molecules, we would then have a coupling strength of $\hbar g_{0} \sqrt{N_{m}}=3.8 \mathrm{meV}$. This coupling is much smaller than both the spectral width of the cavity $(200 \mathrm{meV})$ and the vibrational energy of the molecular vibration considered here $(197 \mathrm{meV})$; therefore, for such small values of collective coupling, we can apply a linearization procedure and obtain the corresponding laser power thresholds for superlinear behavior. Such an estimation, however, provides threshold values for the critical laser intensity that are $N_{m}$ times smaller than those corresponding to the single-molecule coupling. The experimental threshold found in our NPoMs under pulsed illumination [Fig. 2(c) of the main text] matches the estimated collective single-photon coupling $g_{0}$ with $N_{m} \approx 220$, supporting our previous assertion that the coherent coupling between molecular vibrations in distinct molecules is observed. The overestimate in $g_{0}$ (by around $50 \%$ ) is likely due to the dependence on the estimated Raman activity, which is notoriously difficult to calculate for molecules positioned near metallic interfaces. Inaccuracies in $g_{0}$ can also arise from the estimate of the mode volume used.

\section{APPENDIX J: OPTOMECHANICAL DYNAMICS UNDER PULSED ILLUMINATION}

In this section, we discuss the relationship between the characteristics of the SERS system observed under CW and pulsed illumination. The length of the optical pulses (approximately $1 \mathrm{ps)} \mathrm{falls} \mathrm{in} \mathrm{between} \mathrm{the} \mathrm{temporal} \mathrm{char-}$ acteristics of the evolution of plasmons and phonons. In 

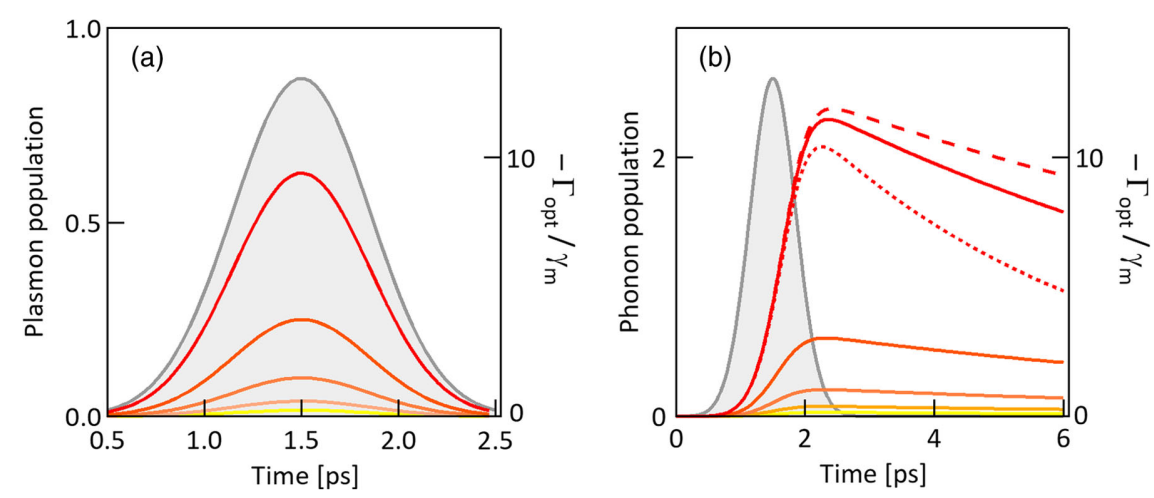

FIG. 14. Evolution of the optomechanical SERS system excited by a 1-ps Gaussian pulse. (a) Evolution of plasmon population for five peak intensities of the incident laser, logarithmically increasing from $6 \times 10^{2} \mu \mathrm{W} / \mu \mathrm{m}^{2}$ (yellow line) to $2.5 \times 10^{4} \mu \mathrm{W} / \mu \mathrm{m}^{2}$ (red line). The shaded area shows normalized optomechanical damping $-\Gamma_{\mathrm{opt}}(t) / \gamma_{m}$, proportional to the instantaneous intensity of the incident pulse. $\Gamma_{\mathrm{opt}}(t)$ is negative, indicating that the system operates in the amplification regime. The plasmonic cavity reacts almost instantaneously to the coherent pump, with the plasmon population closely following the shape of the incident pulse. (b) Phonon population calculated in the same setup, obtained by solving the phonon rate equations. For the largest incident laser intensity, we consider three values of the free-space phonon decay rate: $\hbar \gamma_{m}=0.07 \mathrm{meV}$ is used for all the solid lines, while $\hbar \gamma_{m}=0.14 \mathrm{meV}$ (dotted) and $\hbar \gamma_{m}=0.045 \mathrm{meV}$ (dashed) are also plotted. In all the cases, the buildup of phonons is much slower than that of plasmons, as the vibrations do not reach a steady state during the duration of the pulse. All the remaining parameters are as discussed in the text.

Fig. 14, we show the evolution of plasmon and phonon populations as the system (which remains in thermodynamic equilibrium with the environment) is illuminated by a coherent 1-ps Gaussian pulse. The populations of phonons are calculated by solving the relevant rate equations derived from the linearized master equation given in the main text. On the other hand, the population of plasmons $n_{\mathrm{pl}}$ is calculated by a rate equation accounting only for the coherent pumping and cavity decay:

$$
\frac{d}{d t} \alpha(t)=(-i \Delta-\kappa / 2) \alpha(t)+A(t) .
$$

This description is correct for the weakly coupled optomechanical system with the hierarchy of lifetimes as in molecular optomechanics.

From the results in Fig. 14, it is clear that the vibrations of the molecule will not be able to reach a steady state when illuminated by a 1-ps pulse, irrespective of its temporal shape. However, the effect of reducing the decay rate of phonons, which leads to the nonlinearities observed in the S-SERS experiments, does not require the vibrations to reach the steady state. In fact, as we discuss elsewhere in detail [40], this effect arises from the modification of the Stokes and anti-Stokes transition rates due to the coupling of the molecule to the plasmonic cavity. A convenient description of this modification of the phonon transition rates, expressed through $\Gamma_{\text {eff }}$, requires only that the plasmonic cavity remains in a coherent state.

We note that the largest peak intensities yielding the peak optomechanical damping $-\Gamma_{\text {opt }}(t) / \gamma_{m}>1$ describe a situation in which phonons are generated faster than they decay. In consequence, in the $\mathrm{CW}$ case, the system reaches instability. However, for pulsed illumination, this effect simply accelerates the phonon buildup, but the system remains stable [Fig. 2(b)]. In this case, the free-space phonon decay rate $\gamma_{m}$ does not govern the onset of the nonlinearity, as the buildup of the phonon population over the pulse length is determined by the effective pumping $g_{0} A(t)$, rather than the steady-state expression $\Gamma_{\text {opt }} /\left(\Gamma_{\text {opt }}+\gamma_{m}\right)$ [see Eq. (2)]. We illustrate this effect by comparing the phonon populations for the largest peak laser intensity in Fig. 14(b) calculated for the free-space phonon decay rate $\hbar \gamma_{m}=0.07 \mathrm{meV}$ (red solid line), $\hbar \gamma_{m}=$ $0.14 \mathrm{meV}$ (red dotted line), and $\hbar \gamma_{m}=0.045 \mathrm{meV}$ (red dashed line). The lines diverge only after the pulse begins to decay, and therefore, they have little effect on the overall intensity of the Raman emission. This observation suggests interesting probes more generally of optomechanical systems under pulsed illumination.

\section{APPENDIX K: ESTIMATION OF LOCAL TEMPERATURE WITHIN THE PLASMONIC NANOGAP}

To understand fully the thermal state of the system, local temperature changes are extracted from antiStokes spectra measured at room temperature on single NPoMs [Fig. 15(a)]. The gold electron temperature (presumed equilibrated to the lattice) is estimated by fitting the anti-Stokes background to $I_{\mathrm{AS}}^{\mathrm{bgd}}(\Delta \omega) \propto[\exp (\hbar \Delta \omega /$ $\left.\left.k_{B} T\right)-1\right]^{-1}$ for a frequency difference $\Delta \omega$ from the pump $\omega_{l}$. The power regime where SERS increases linearly 

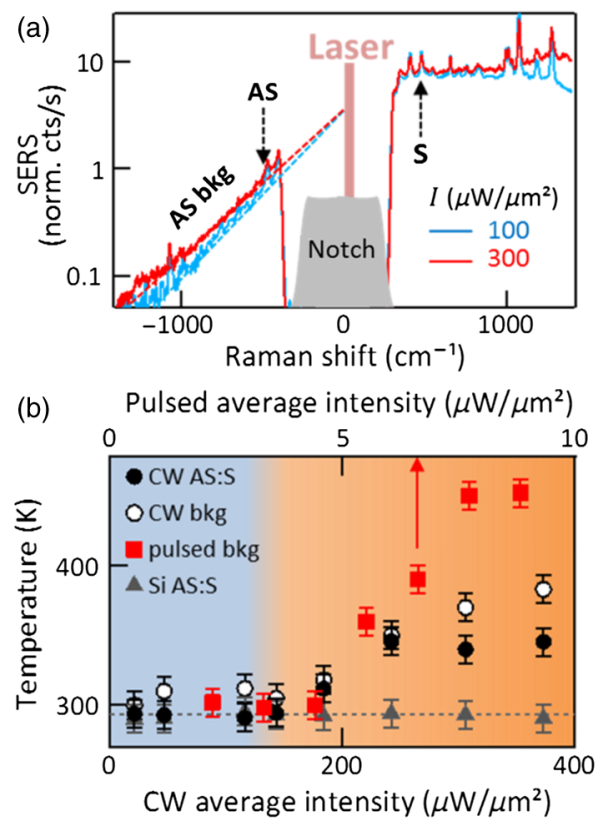

FIG. 15. (a) SERS intensities from a single NPoM at $100 \mu \mathrm{W} / \mu \mathrm{m}^{2}$ (blue curve) and $300 \mu \mathrm{W} / \mu \mathrm{m}^{2}$ (red curve) 633-nm CW excitation, normalized to a $475-\mathrm{cm}^{-1}$ AS peak. Stokes and corresponding anti-Stokes peaks used in calculations are highlighted with arrows; anti-Stokes background fits are dashed lines. (b) Local temperature increase vs laser intensity for both CW (lower axis, circles) and pulsed (upper axis, squares) excitation. Temperature is extracted from the Stokes to antiStokes ratio (CW-black circles) or from the anti-Stokes background (white circles for CW, red squares for pulsed). Reference temperatures from the bulk silicon are shown in grey. Shading shows linear (blue) and nonlinear (orange) Stokes regimes in the pulsed excitation case.

[Fig. 15(b) blue region $R_{L}$ ] shows a negligible temperature increase for both $\mathrm{CW}$ (white circles) and pulsed (red squares) excitation. By contrast, in the nonlinear $R_{\mathrm{NL}}$ regime [Fig. 15(b) orange region], a linear temperature increase is observed, reaching $380 \mathrm{~K}(\mathrm{CW})$ and $420 \mathrm{~K}$ (pulsed) for $300 \mu \mathrm{W} / \mu \mathrm{m}^{2}$ excitation. A different "molecular temperature" within the plasmonic nanogap is extracted from the anti-Stokes/Stokes ratio of the strongest line (Fig. 15 arrows), using [8,20,40]

$$
\frac{I_{\mathrm{AS}}}{I_{S}}=\left(\frac{\omega_{l}+\Delta \omega}{\omega_{l}-\Delta \omega}\right)^{4} \times \exp \left(-\frac{\hbar \Delta \omega}{k_{B} T_{\mathrm{eff}}}\right)
$$

for the CW case (Fig. 15, black points), which yields the clearest emission peaks. $T_{\text {eff }}$ is the effective temperature of the vibrational modes and can differ from the actual sample temperature $T$. This marked temperature increase for $>150 \mu \mathrm{W} / \mu \mathrm{m}^{2}$ laser intensities is consistent with previous literature [60-63] and thought to be from local heating of the substrate and analyte due to light absorption in the metal [64]. This thermal heating is unable to account for the nonlinear increase in pulsed Stokes emission (see Appendix L).

The anti-Stokes molecular signals for the phonon occupations here are predicted to be $>100$-fold weaker than the Stokes emission, making them difficult to see in the pulsed regime except for very long integration times, which can lead to degradation of the NPoM structure.

Such data in Fig. 16 show that the ratio of Stokes to anti-Stokes is approximately 160 near the highest powers of the superlinear regime. Comparing to Fig. 2(b) shows this is in line with that expected from the optomechanical model.

\section{APPENDIX L: ESTIMATION OF HEATING CONTRIBUTIONS TO S-SERS}

We will explore an alternative explanation to molecular optomechanics for the superlinear dependence of the Stokes intensity on the peak laser power, in which the nonlinearity is instead due to the strong localized heating of the molecule by the laser, leading to the onset of phononstimulated Stokes emission, rather than the vanishing of the effective decay rate. In that case, the deviation of the power dependence of the Stokes emission follows $S_{S} \propto I\left(1+n_{\text {th }}\right)$. Notably, we neglect any contribution from the vibrational pumping, as well as the change in the effective phonon damping rate. Consequently, by investigating the superlinear growth of the Stokes emission, we can estimate the effective molecular temperature. For the points marked in Fig. 2(c) with red circles, the extracted temperatures are given in Fig. 17. As the derived temperatures would need to be in excess of $4000 \mathrm{~K}$, we conclude

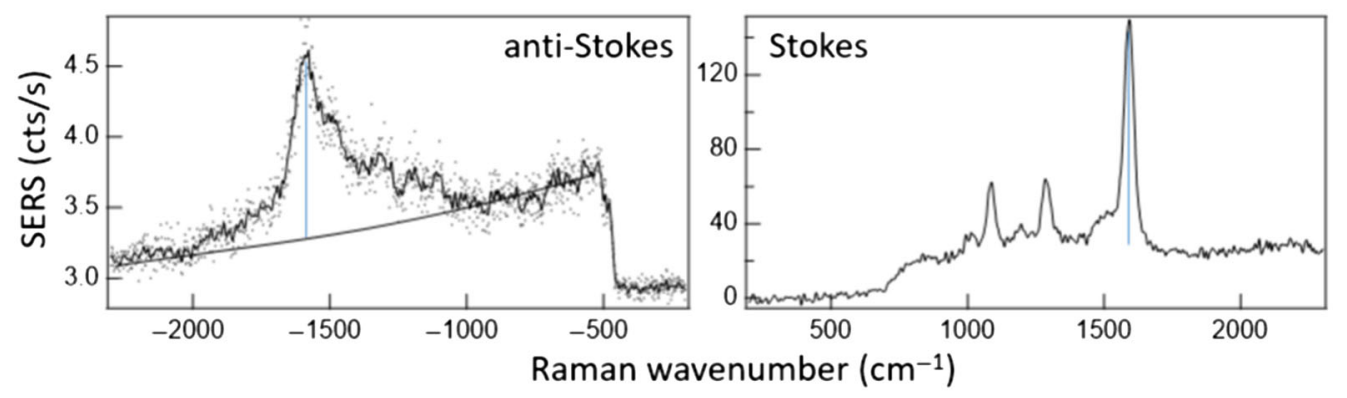

FIG. 16. Comparison of pulsed SERS from single BPT NPoM for anti-Stokes and Stokes contributions in the nonlinear regime. 


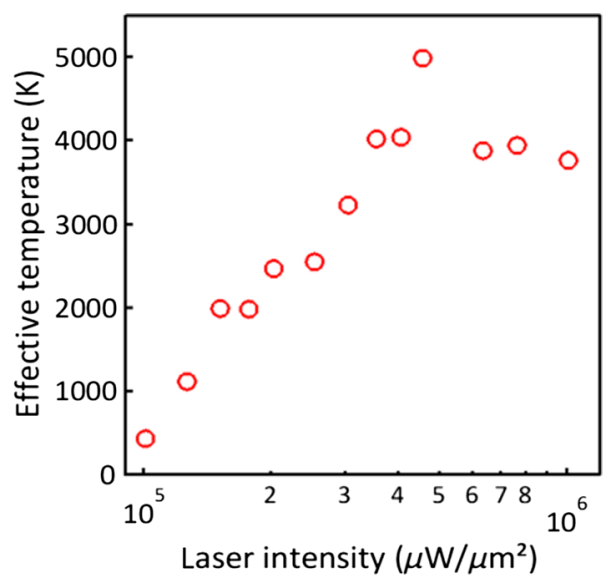

FIG. 17. Estimation of the effective temperature from the Stokes intensity under pulsed illumination for the data shown with red circles in Fig. 2(c), when optomechanical interactions are not accounted for. Unrealistically high temperatures are obtained in such a case.

that the superlinear Stokes signal cannot be explained as an emission stimulated by the incoherent population of thermal phonons.

[1] F. M. Hoffmann, Infrared Reflection-Absorption Spectroscopy of Adsorbed Molecules, Surf. Sci. Rep. 3, 107 (1983).

[2] D. J. Nesbitt, High-Resolution Infrared Spectroscopy of Weakly Bound Molecular Complexes, Chem. Rev. 88, 843 (1988).

[3] S. Nie and S. R. Emory, Probing Single Molecules and Single Nanoparticles by Surface-Enhanced Raman Scattering, Science 275, 1102 (1997).

[4] K. Kneipp, H. Kneipp, I. Itzkan, R. R. Dasari, and M. S. Feld, Ultrasensitive Chemical Analysis by Raman Spectroscopy, Chem. Rev. 99, 2957 (1999).

[5] X. Zhang, Q.H. Tan, J. B. Wu, W. Shi, and P.H. Tan, Review on the Raman Spectroscopy of Different Types of Layered Materials, Nanoscale 8, 6435 (2016).

[6] K. Kneipp, Y. Wang, H. Kneipp, L. T. Perelman, I. Itzkan, R. R. Dasari, and M.S. Feld, Single Molecule Detection Using Surface-Enhanced Raman Scattering (SERS), Phys. Rev. Lett. 78, 1667 (1997).

[7] J. P. Gordon, H. J. Zeiger, and C. H. Townes, The MaserNew Type of Microwave Amplifier, Frequency Standard, and Spectrometer, Phys. Rev. 99, 1264 (1955).

[8] F. Benz, M. K. Schmidt, A. Dreismann, R. Chikkaraddy, Y. Zhang, A. Demetriadou, C. Carnegie, H. Ohadi, B. de Nijs, R. Esteban, J. Aizpurua, and J.J. Baumberg, SingleMolecule Optomechanics in "Picocavities", Science 354, 726 (2016).

[9] H. Okamoto, T. Watanabe, R. Ohta, K. Onomitsu, H. Gotoh, T. Sogawa, and H. Yamaguchi, Cavity-Less On-Chip Optomechanics Using Excitonic Transitions in Semiconductor Heterostructures, Nat. Commun. 6, 8478 (2015).
[10] A. Schmidsfeld and M. Reichling, Controlling the OptoMechanics of a Cantilever in an Interferometer via Cavity Loss, Appl. Phys. Lett. 107, 123111 (2015).

[11] K. E. Grutter, M. I. Davanco, and K. Srinivasan, Slot-Mode Optomechanical Crystals: A Versatile Platform for Multimode Optomechanics, Optica 2, 994 (2015).

[12] A. Schliesser, R. Riviere, G. Anetsberger, O. Arcizet, and T. J. Kippenberg, Resolved-Sideband Cooling of a Micromechanical Oscillator, Nat. Phys. 4, 415 (2008).

[13] S. Weis, R. Riviere, S. Deleglise, E. Gavartin, E. Arcizet, A. Schliesser, and T. J. Kippenberg, Optomechanically Induced Transparency, Science 330, 1520 (2010).

[14] O. Graydon, Optomechanics: Enhanced Cavities, Nat. Photonics 7, 167 (2013).

[15] A. G. Kuhn, M. Bahriz, O. Ducloux, C. Chartier, O. Le Traon, T. Briant, P. F. Cohadon, A. Heidmann, C. Michel, L. Pinard, and R. A. Flaminio, Micropillar for Cavity Optomechanics, Appl. Phys. Lett. 99, 121103 (2011).

[16] J. Chan, T.P. Mayer Alegre, A. H. Safavi-Naeini, J. T. Hill, A. Krause, S. Groblacher, M. Aspelmeyer, and O. Painter, Laser Cooling of a Nanomechanical Oscillator into its Quantum Ground State, Nature (London) 478, 89 (2011).

[17] J. Zheng, Y. Li, M. S. Aras, A. Stein, K. L. Shepard, and C. W. Wong, Parametric Optomechanical Oscillations in Two-Dimensional Slot-Type High-Q Photonic Crystal Cavities, Appl. Phys. Lett. 100, 211908 (2012).

[18] A. Butsch, R. Koehler, R. E. Noskov, and P. S. J. Russel, CW-Pumped Single-Pass Frequency Comb Generation by Resonant Optomechanical Nonlinearity in Dual-Nanoweb Fiber, Optica 1, 158 (2014).

[19] P. Roelli, C. Galland, N. Piro, and T. J. Kippenberg, Molecular Cavity Optomechanics as a Theory of Plasmon-Enhanced Raman Scattering, Nat. Nanotechnol. 11, 164 (2016).

[20] M. K. Schmidt, R. Esteban, A. Gonzalez-Tudela, G. Giedke, and J. Aizpurua, Quantum Mechanical Description of Raman Scattering from Molecules in Plasmonic Cavities, ACS Nano 10, 6291 (2016).

[21] R. Zhang, Y. Zhang, Z. Dong, S. Jiang, C. Zhang, L. Chen, L. Zhang, Y. Liao, J. Aizpurua, Y. Luo, J. Yang, and J. Hou, Chemical Mapping of a Single Molecule by PlasmonEnhanced Raman Scattering, Nature (London) 498, 82 (2013).

[22] T. J. Kippenberg, H. Rokhsari, T. Carmon, A. Scherer, and K. J. Vahala, Analysis of Radiation-Pressure Induced Mechanical Oscillation of an Optical Microcavity, Phys. Rev. Lett. 95, 033901 (2005).

[23] T. Carmon, H. Rokhsari, L. Yang, T. J. Kippenberg, and K. J. Vahala, Temporal Behavior of Radiation PressureInduced Vibrations of an Optical Microcavity Phonon Mode, Phys. Rev. Lett. 94, 223902 (2005).

[24] F. Marquardt, J. G. E. Harris, and S. M. Girvin, Dynamical Multistability Induced by Radiation Pressure in HighFinesse Micromechanical Optical Cavities, Phys. Rev. Lett. 96, 103901 (2006).

[25] K. Kneipp, Y. Wang, H. Kneipp, L. T. Perelman, I. Itzkan, R. R. Dasari, and M.S. Feld, Single Molecule Detection Using Surface-Enhanced Raman Scattering (SERS), Phys. Rev. Lett. 78, 1667 (1997). 
[26] H. Xu, E. J. Bjerneld, M. Kall, and L. Borjesson, Spectroscopy of Single Haemoglobin Molecules by Surface Enhanced Raman Scattering, Phys. Rev. Lett. 83, 4357 (1999).

[27] P. L. Stiles, J. A. Dieringer, N. C. Shah, and R. P. Van Duyne, Surface-Enhanced Raman Spectroscopy, Annu. Rev. Anal. Chem. 1, 601 (2008).

[28] A. Lombardi, A. Demetriadou, L. Weller, P. Andrae, F. Benz, R. Chikkaraddy, J. Aizpurua, and J. J. Baumberg, Anomalous Spectral Shift of Near-and Far-Field Plasmonic Resonances in Nanogaps, ACS Photonics 3, 471 (2016).

[29] J. C. Love, L. A. Estroff, J. K. Kriebel, R. G. Nuzzo, and G. M. Whitesides, Self-Assembled Monolayers of Thiolates on Metals as a Form of Nanotechnology, Chem. Rev. 105, 1103 (2005).

[30] W. Zhu and K. B. Crozier, Quantum Mechanical Limit to Plasmonic Enhancement as Observed by Surface-Enhanced Raman Scattering, Nat. Commun. 5, 5228 (2014).

[31] X. S. Zheng, P. Hu, J. H. Zhong, C. Zong, X. Wang, B. J. Liu, and B. Ren, Laser Power Dependent Surface-Enhanced Raman Spectroscopic Study of 4-Mercaptopyridine on Uniform Gold Nanoparticle-Assembled Substrates, J. Phys. Chem. C 118, 3750 (2014).

[32] Y. Kitahama, Y. Tanaka, T. Itoh, and Y. Ozaki, Power-Law Analysis of Surface-Plasmon-Enhanced Electromagnetic Field Dependence of Blinking SERS of Thiacyanine or Thiacarbocyanine Adsorbed on Single Silver Nanoaggregates, Phys. Chem. Chem. Phys. 13, 7439 (2011).

[33] L. Kang, P. Xu, B. Zhang, H. Tsai, X. Han, and H. L. Wang, Laser Wavelength- and Power-Dependent Plasmon-Driven Chemical Reactions Monitored Using Single Particle Surface Enhanced Raman Spectroscopy, Chem. Commun. (Cambridge) 49, 3389 (2013).

[34] K. T. Crampton, A. Zeytunyan, A. S. Fast, F. T. Ladani, A. Alfonso-Garcia, M. Banik, S. Yampolsky, D. A. Fishman, E. O. Potma, and V. Ara Apkarian, Ultrafast Coherent Raman Scattering at Plasmonic Nanojunctions, J. Phys. Chem. C 120, 20943 (2016).

[35] J. K. Daniels and G. Chumanov, Nanoparticle-Mirror Sandwich Substrates for Surface-Enhanced Raman Scattering, J. Phys. Chem. B 109, 17936 (2005).

[36] S. Mubeen, S. Zhang, N. Kim, S. Lee, S. Kramer, H. Xu, and M. Moskovits, Plasmonic Properties of Gold Nanoparticles Separated from a Gold Mirror by an Ultrathin Oxide, Nano Lett. 12, 2088 (2012).

[37] C. Tserkezis, R. Esteban, D. O. Sigle, J. Mertens, L. O. Herrmann, J. J. Baumberg, and J. Aizpurua, Hybridization of Plasmonic Antenna and Cavity Modes: Extreme Optics of Nanoparticle-on-Mirror Nanogaps, Phys. Rev. A 92, 053811 (2015).

[38] R. Chikkaraddy, B. de Nijs, F. Benz, S. J. Barrow, O. A. Scherman, E. Rosta, A. Demetriadou, P. Fox, O. Hess, and J. J. Baumberg, Single-Molecule Strong Coupling at Room Temperature in Plasmonic Nanocavities, Nature (London) 535, 127 (2016).

[39] F. Benz, C. Tserkezis, L. O. Herrmann, B. De Nijs, A. Sanders, D. O. Sigle, L. Pukenas, S. D. Evans, J. Aizpurua, and J. J. Baumberg, Nanooptics of Molecular-Shunted Plasmonic Nanojunction, Nano Lett. 15, 669 (2015).
[40] M. K. Schmidt, R. Esteban, F. Benz, J. J. Baumberg, and J. Aizpurua, Linking Classical and Molecular Optomechanics Descriptions of SERS, Faraday Discuss. 205, 31 (2017).

[41] M. Kamandar Dezfouli and S. Hughes, Quantum Optics Model of Surface-Enhanced Raman Spectroscopy for Arbitrarily Shaped Plasmonic Resonators, ACS Photonics 4, 1245 (2017).

[42] H.-H. Shin, G. Jin Yeon, H.-K. Choi, S.-M. Park, K. Sup Lee, and Z. Hwan Kim, Frequency-Domain Proof of the Existence of Atomic-Scale SERS Hot-Spots, Nano Lett. (2017).

[43] J. T. Hugall and J. J. Baumberg, Demonstrating Photoluminescence from Au Is Electronic Inelastic Light Scattering of a Plasmonic Metal: The Origin of SERS Background, Nano Lett. 15, 2600 (2015).

[44] G. Smith, J.-S. Girardon, J.-F. Paul, and E. Berrier, Dynamics of a Plasmon-Activated p-Mercaptobenzoic Acid Layer Deposited over Au Nanoparticles Using Time-Resolved SERS, Phys. Chem. Chem. Phys. 18, 19567 (2016).

[45] J. Long, H. Yi, H. Li, Z. Lei, and T. Yang, Reproducible Ultrahigh SERS Enhancement in Single Deterministic Hotspots Using Nanosphere-Plane Antennas Under Radially Polarized Excitation, Sci. Rep. 6, 33218 (2016).

[46] K.-D. Park, E. A. Muller, V. Kravtsov, P. M. Sass, J. Dreyer, J. M. Atkin, and M. B. Raschke, Variable-Temperature Tip-Enhanced Raman Spectroscopy of Single-Molecule Fluctuations and Dynamics, Nano Lett. 16, 479 (2016).

[47] D. O. Sigle, J. Mertens, L. O. Herrmann, R. W. Bowman, S. Ithurria, B. Dubertret, Y. Shi, H. Y. Yang, C. Tserkezis, J. Aizpurua, and J. J. Baumberg, Monitoring Morphological Changes in 2D Monolayer Semiconductors Using AtomThick Plasmonic Nanocavities, ACS Nano 9, 825 (2015).

[48] H. Häkkinen, The Gold Sulfur Interface at the Nanoscale, Nat. Chem. 4, 443 (2012).

[49] T. Bürgi, Properties of the Gold-Sulphur Interface: From Self-Assembled Monolayers to Clusters, Nanoscale 7, 15553 (2015).

[50] P. Christopher, H. Xin, A. Marimuthu, and S. Linic, Singular Characteristics and Unique Chemical Bond Activation Mechanisms of Photocatalytic Reactions on Plasmonic Nanostructures, Nat. Mater. 11, 1044 (2012).

[51] D. B. Ingram and S. Linic, Water Splitting on Composite Plasmonic-Metal/Semiconductor Photoelectrodes: Evidence for Selective Plasmon-Induced Formation of Charge Carriers near the Semiconductor Surface, J. Am. Chem. Soc. 133, 5202 (2011).

[52] M. L. Brongersma, N. J. Halas, and P. Nordlander, PlasmonInduced Hot Carrier Science and Technology, Nat. Nanotechnol. 10, 25 (2015).

[53] E. M. van Schrojenstein Lantman, T. Deckert-Gaudig, A. J. G. Mank, V. Deckert, and B. M. Weckhuysen, Catalytic Processes Monitored at the Nanoscale with Tip-Enhanced Raman Spectroscopy, Nat. Nanotechnol. 7, 583 (2012).

[54] M. Kornbluth, A. Nitzan, and T. Seideman, Light-Induced Electronic Non-Equilibrium in Plasmonic Particles, J. Chem. Phys. 138, 174707 (2013).

[55] M. Hegner, P. Wagner, and G. Semenza, Ultrafast Atomically Flat Template-Stripped Au Surfaces for Scanning Probe Microscopy, Surf. Sci. 291, 39 (1993). 
[56] F. Verluise, V. Laude, Z. Cheng, C. Spielmann, and P. Tournois, Amplitude and Phase Control of Ultrashort Pulses by Use of an Acousto-Optic Programmable Dispersive Filter: Pulse Compression and Shaping, Opt. Lett. 25, 575 (2000).

[57] R. W. Taylor, F. Benz, D. O. Sigle, R. W. Bowman, P. Bao, J. S. Roth, G. R. Heath, S. D. Evans, and J. J. Baumberg, Watching Individual Molecules Flex within Lipid Membranes Using SERS, Sci. Rep. 4, 5490 (2014).

[58] W. Geyer, V. Stadler, W. Eck, M. Zharnikov, A. Gölzhäuser, and M. Grunze, Electron-Induced Crosslinking of Aromatic Self-Assembled Monolayers: negative Resists for Nanolithography, Appl. Phys. Lett. 75, 2401 (1999).

[59] R. Esteban, J. Aizpurua, and G. W. Bryant, Strong Coupling of Single Emitters Interacting with Phononic Infrared Antennae, New J. Phys. 16, 013052 (2014).

[60] R. R. Letfullin, T. F. George, G. C. Duree, and B. M. Bollinger, Ultrashort Laser Pulse Heating of Nanopar- ticles: Comparison of Theoretical Approaches, Adv. Opt. Technol. 2008, 251718 (2008).

[61] Z. Qin and J. C. Bischof, Thermophysical and Biological Responses of Gold Nanoparticle Laser Heating, Chem. Soc. Rev. 41, 1191 (2012).

[62] R. C. Maher, L. F. Cohen, J. C. Gallop, E. C. Le Ru, and P. G. Etchegoin, Temperature-Dependent Anti-Stokes/ Stokes Ratios under Surface-Enhanced Raman Scattering Conditions, J. Phys. Chem. B 110, 6797 (2006).

[63] E. A. Pozzi, A. B. Zrimsek, C. M. Lethiec, G. C. Schatz, M. C. Hersam, and R.P. Van Duyne, Evaluating SingleMolecule Stokes and Anti-Stokes SERS for Nanoscale Thermometry, J. Phys. Chem. C 119, 21116 (2015).

[64] A. O. Govorov and H. H. Richardson, Generating Heat with Metal Nanoparticles, Nano Today 2, 30 (2007). 